\title{
Magnetotransport and internodal tunnelling in Weyl semimetals
}

\author{
G. Bednik, ${ }^{1}$ K. S. Tikhonov, ${ }^{2,3}$ and S. V. Syzranov $\oplus^{1}$ \\ ${ }^{1}$ Physics Department, University of California, Santa Cruz, California 95064, USA \\ ${ }^{2}$ L. D. Landau Institute for Theoretical Physics, 119334 Moscow, Russia \\ ${ }^{3}$ Condensed-matter Physics Laboratory, National Research University Higher School of Economics, 101000 Moscow, Russia
}

(Received 18 December 2019; accepted 7 April 2020; published 4 May 2020)

\begin{abstract}
Internodal dynamics of quasiparticles in Weyl semimetals manifest themselves in hydrodynamic, transport, and thermodynamic phenomena and are essential for potential valleytronic applications of these systems. In an external magnetic field, coherent quasiparticle tunnelling between the nodes modifies the quasiparticle dispersion and, in particular, opens gaps in the dispersion of quasiparticles at the zeroth Landau level. We study magnetotransport in a Weyl semimetal taking into account mechanisms of quasiparticle scattering, both affected by such gaps and independent of them. We compute the longitudinal resistivity of a disordered Weyl semimetal with two nodes in a strong magnetic field microscopically and demonstrate that in a broad range of magnetic fields, it has a strong angular dependence, $\rho(\eta) \propto C_{1}+C_{2} \cos ^{2} \eta$, where $\eta$ is the angle between the field and the separation between the nodes in momentum space. The first term is determined by the coherent internodal tunnelling and is important only at angles $\eta$ close to $\pi / 2$. This contribution depends exponentially on the magnetic field, $\propto \exp \left(-B_{0} / B\right)$. The second term is weakly dependent on the absolute value of the magnetic field for realistic concentrations of the impurities in a broad interval of fields.
\end{abstract}

DOI: 10.1103/PhysRevResearch.2.023124

\section{INTRODUCTION}

Recent prediction [1] and experimental discovery [2-9] of quasiparticles with Weyl dispersion in solid-state systems have motivated a vast number of predictions and observations of novel fundamental effects involving Weyl particles (see, e.g., Refs. $[10,11]$ for a review). Some of these phenomena, such as the chiral anomaly [12-14], rely on the transfer of quasiparticles between different Weyl nodes, sometimes also referred to as valleys, i.e., points in momentum space in whose vicinity the quasiparticles display Weyl dispersion.

Weyl nodes in solid-state systems come in pairs with opposite chiralities [15]. Apart from fundamental interest, internodal dynamics of Weyl quasiparticles may be used for valleytronic applications, i.e., using the valley degree of freedom to store, process, and transfer information and to control electron transport [16]. Experimentally observed manifestations of the internodal (intervalley) dynamics also include negative longitudinal magnetoresistance (see, e.g., Refs. [14,17-25]), a consequence of the chiral anomaly $[12,15]$, and quantum oscillations of resistance in thin slabs of Weyl semimetals (WSMs) [26,27]. Changing the valley degree of freedom of quasiparticles has also been predicted to lead to the release or absorption of heat ("adiabatic dechiralization" [28]) and to affect the hydrodynamic flows of electrons in WSMs [29-36].

Published by the American Physical Society under the terms of the Creative Commons Attribution 4.0 International license. Further distribution of this work must maintain attribution to the author(s) and the published article's title, journal citation, and DOI.
Of fundamental importance for valleytronic applications is coherent tunnelling between Weyl nodes. Such tunnelling leads to an effective coupling between the states of quasiparticles near different nodes, which may be controlled by the direction and the magnitude of an external magnetic field. This coupling leads to the opening of a gap in the quasiparticle dispersion at the zeroth Landau level in WSMs [37-39], which has recently been observed in experiment [40-42].

In this paper, we study the interplay of magnetotransport in disordered Weyl semimetals and coherent internodal tunnelling. We demonstrate that the gap $2 \Delta$, created in the quasiparticle dispersion by such tunnelling, significantly affects the longitudinal conduction (conduction along the magnetic field) of the quasiparticles if the magnetic field is perpendicular to the line connecting a pair of nodes in momentum space. Quasiparticle states are strongly hybridized between the nodes in this regime, and their scattering is affected by long-range correlated disorder, with the correlation length longer than the inverse separation between the nodes in momentum space. The longitudinal resistivity in this regime depends exponentially on the magnitude of the magnetic field.

For the other directions of the magnetic field, the internodal hybridization of the quasiparticle states may be neglected. The resistivity is then determined by large-momentum scattering between states at different nodes and has a strong dependence on the direction of the field.

Our results demonstrate how the internodal coupling (the gaps in the spectra of Landau levels) in Weyl semimetals may be observed in transport experiments. Furthermore, the strong dependence of the internodal coupling on the external magnetic field can be used in valleytronic devices to control electron transport if the magnetic field is perpendicular to the separation between the nodes. 
The paper is organized as follows. In Sec. II, we summarize our results for the magnetoconductance of a Weyl semimetal in a magnetic field. A detailed microscopic discussion of the model of a disordered two-node Weyl semimetal and its typical parameters is presented in Sec. III. We discuss the quasiparticle dispersion in the system in Sec. IV. Sections V and VI deal with quasiparticle scattering off impurities and magnetoconductance in disordered semimetals. In Sec. V, we consider generic directions of the magnetic field where the magnetoresistance is weakly affected by the internodal coupling. Finally, in Sec. VI, we demonstrate that the conduction is strongly affected by the coupling if the magnetic field is perpendicular to the line connecting the nodes, and compute the resistivity in this regime.

\section{RESULTS}

In the presence of an external magnetic field in a sufficiently clean system, the quasiparticle motion is quantized in the plane perpendicular to the field. At the zeroth Landau level, quasiparticles at each Weyl node may move only in one direction, i.e., either parallel or antiparallel to the magnetic field, depending on the chirality of the node, while quasiparticles at higher Landau levels may move both parallel and antiparallel to the magnetic field.

As a result, at strong magnetic fields or low levels of doping, for which only the zeroth Landau level contributes to transport (the ultraquantum limit), the longitudinal resistivity of a Weyl semimetal is determined by the internodal scattering. There are two main mechanisms of such internodal scattering: (1) large-momentum scattering between states at different nodes by impurities and (2) the interplay of smallmomentum processes near one node and the internodal hybridization of quasiparticle states.

In this paper, we analyze the dependence of the magnetoconductivity in a Weyl semimetal with two nodes as a function of the magnetic field $B$ and its direction, focusing on the interplay of the internodal tunnelling and magnetoresistance. In the ultraquantum limit, the longitudinal resistivity of a Weyl semimetal with charged impurities may be described by the interpolation formula

$$
\rho(\eta, \mathbf{B})=\rho_{\text {inter }} \cos ^{2} \eta+\rho_{\text {gap }}(B, \eta),
$$

where $\rho_{\text {inter }} \cos ^{2} \eta$ is the contribution of large-momentum elastic scattering and is weakly dependent on the absolute value $B$ of the magnetic field; the last term $\rho_{\text {gap }}(B, \eta)$ accounts for the effects of the gap in the quasiparticle dispersion at the zeroth Landau level caused by coherent internodal tunnelling; $\eta$ is the angle between the field and the separation between the nodes in momentum space.

For screened charged impurities, ubiquitous in Weyl and semiconducting systems, the function $\rho_{\text {inter }}$ weakly depends on the absolute value $B$ of the magnetic field in the experimentally important limit $l_{B} Q \gg 1$, where $l_{B}$ is the magnetic length and $2 Q$ is the momentum separation between the nodes. Magnetoresistance independent of the absolute value of the magnetic field has also been demonstrated previously (see, e.g., Refs. [43-45]) for the special case of Gaussian impurities of the width $a \ll l_{B}$. For the magnetic fields corresponding to $l_{B} Q \gg 1$ (the limit opposite to the one considered here), a
Weyl semimetal with charged impurities exhibits a different dependence of the resistivity $\left(\rho_{\text {inter }} \propto 1 / B^{2}\right)$ on the magnitude of the magnetic field [44].

In this paper, we also find a strong angular dependence $\propto \cos ^{2} \eta$ of the first contribution $\rho_{\text {inter }}$ to the resistivity (2.1). Such anisotropic behavior of the resistivity comes the structure of the wave functions at the nodes.

The second contribution, $\rho_{\text {gap }}(B, \eta)$, is suppressed in the experimentally important case of weak magnetic fields and is, therefore, important at angles close to $\eta=\pi / 2$. This contribution exhibits an exponential dependence,

$$
\rho_{\text {gap }}(B, \pi / 2) \propto \exp \left(-B_{0} / B\right),
$$

on the magnitude of the magnetic field, where the characteristic field $B_{0}$ is given by the integral

$$
B_{0}=\frac{2 c}{|e| v_{F} \hbar} \int_{\text {node } 1}^{\text {node } 2} \xi_{\mathbf{k}} d k
$$

of the quasiparticle dispersion $\xi_{\mathbf{k}}$ along the minimum-action tunnelling path between the nodes in momentum space; $v_{F}$ is the Fermi velocity which is assumed to be isotropic near each node. The exponential dependence of the resistance (2.1) on the magnetic field comes from the existence of the gap [39] in the dispersion of the quasiparticles, where the characteristic field $B_{0}$ may be of the order of $10 T$ or larger [39].

Away from the ultraquantum limit, i.e., at higher levels of doping or smaller magnetic fields, nonzero Landau levels contribute to the resistivity. Unlike the zeroth Landau level, quasiparticles at those higher Landau levels can be backscattered within the same node. Because of the long-range-correlated nature of the potential of screened impurities, such intranodal scattering at higher Landau levels is significantly stronger than the internodal scattering. The resistivity in this regime has recently been a subject of numerous studies (see, e.g., Refs. $[12,14,46,47])$ and is not a focus of this paper.

At the levels of doping and magnetic fields where the higher Landau levels contribute to transport, their contribution $\rho_{\text {intra }}(B)$ to the resistivity may be added to the contributions (2.1) on which we focus in this paper. Different contributions may be separated from each other using their different dependencies on the direction and the value of the magnetic field. In the case of isotropic dispersions at Weyl nodes, the contribution $\rho_{\text {intra }}(B)$ of the intranodal scattering at high Landau levels is isotropic, which may be used to separate it from the first term in Eq. (2.1). It also has a weaker than exponential dependence on the magnetic field, which distinguishes it from the effects of the gap at the zeroth Landau level described by the second term in Eq. (2.1). For simplicity, in this paper, we focus on the ultraquantum limit, where the contribution $\rho_{\text {intra }}(B)$ is absent and the full resistivity is, thus, described by Eq. (2.1).

We compute both contributions to the resistivity (2.1) microscopically. We find that for the experimentally important case of charged impurities and sufficiently small magnetic fields,

$$
\rho_{\text {inter }}=\frac{2 \pi^{3} n e^{2}}{v_{F}^{2} Q^{4} \hbar \varepsilon^{2}},
$$


where $n$ is the concentration of the impurities and $\varepsilon$ is the dielectric constant. For the gap-dependent contribution, which for the experimentally important case of low fields is relevant only at $\eta \approx \frac{\pi}{2}$ (or, equivalently, $\eta \approx-\frac{\pi}{2}$ ), we obtain

$$
\rho_{\text {gap }}\left(B, \frac{\pi}{2}\right)=C(B) \frac{n c|e| \hbar^{2}}{\mu_{0}^{2} B_{\mu}^{2}} B \min \left[1,\left(\frac{B}{B_{\mu}}\right)^{3}\right] e^{-\frac{B_{0}}{B}},
$$

where $C(B)$ is a dimensionless coefficient weakly dependent on the magnetic field and where we introduced the characteristic magnetic field

$$
B_{\mu}=\left(\frac{2 \pi}{9 \alpha}\right)^{\frac{1}{3}} \frac{c \mu_{0}^{2}}{|e| v_{F}^{2} \hbar},
$$

where $\alpha=\frac{e^{2}}{\varepsilon \hbar v_{F}}$ is the so-called fine-structure constant in a Weyl semimetal and $\mu_{0}$ is the chemical potential in the system at zero magnetic field (measured from the energy of the Weyl node).

For a semimetal with the chemical potential $\mu_{0}=$ $10 \mathrm{meV}$ (corresponding to the concentration of dopants $n \sim$ $10^{15} \mathrm{~cm}^{-3}$ ) and the dielectric constant [48-51] $\varepsilon=10-50$, the characteristic magnetic field $B_{\mu}$ lies, respectively, in the interval $B_{\mu}=0.2-0.4 T$. Fields of this order of magnitude also correspond to entering the ultraquantum limit for the characteristic chemical potentials under consideration. For such fields, the gap-dependent contribution $\rho_{\text {gap }}(B, \eta)$ is strongly suppressed due to its exponential dependence on the magnetic fields, except for selected directions of the field, corresponding to $\eta= \pm \pi / 2$. Thus, we expect our results for the contributions to Eq. (2.1) to hold in realistic Weyl semimetals for magnetic fields up to several tesla. For stronger fields, the resistivity is dominated by the gap-dependent contribution $\rho_{\text {gap }}(B, \eta)$ and has a strong exponential dependence on the magnetic field for all directions of the magnetic field (except $\eta=0, \pi$, where the gap vanishes).

We emphasise that although in most of this paper we consider a system with a single pair of Weyl nodes, our results may easily be generalized to the case of a system with multiple pairs of nodes, e.g., TaAs or TaP. The conductivity in such a system is given by the sum $\sigma(\mathbf{B})=$ $\sum_{i}\left[\rho_{\text {inter }} \cos ^{2} \eta_{i}+\rho_{\text {gap }}\left(B, \eta_{i}\right)\right]^{-1}$ of contributions from pairs of close nodes, where $\eta_{i}$ is the angle between the field and the difference of momenta of the nodes in the $i$ th pair.

\section{MODEL}

We consider a Weyl semimetal (WSM) with two nodes separated along the $z$ axis in momentum space. The Hamiltonian of the quasiparticles in this system, in the presence of charged impurities, is given by

$$
\begin{aligned}
\hat{H}= & v_{F}\left(\hat{k}_{x}-\frac{e}{c} A_{x}\right) \hat{\sigma}_{x}+v_{F}\left(\hat{k}_{y}-\frac{e}{c} A_{y}\right) \hat{\sigma}_{y} \\
& +\hat{\sigma}_{z} m\left(\hat{k}_{z}-\frac{e}{c} A_{z}\right)+\frac{e^{2}}{\epsilon} \sum_{i} Z_{i} \frac{e^{-\varkappa\left|\mathbf{r}-\mathbf{R}_{i}\right|}}{\left|\mathbf{r}-\mathbf{R}_{i}\right|},
\end{aligned}
$$

where the first three terms in the right-hand side (rhs) represent the kinetic energy of the quasiparticles in a magnetic field and the last term accounts for the potential of screened impurities; $\hat{\mathbf{k}}=\left(\hat{k}_{x}, \hat{k}_{y}, \hat{k}_{z}\right)=-i \partial_{\mathbf{r}}$ is the operator of the

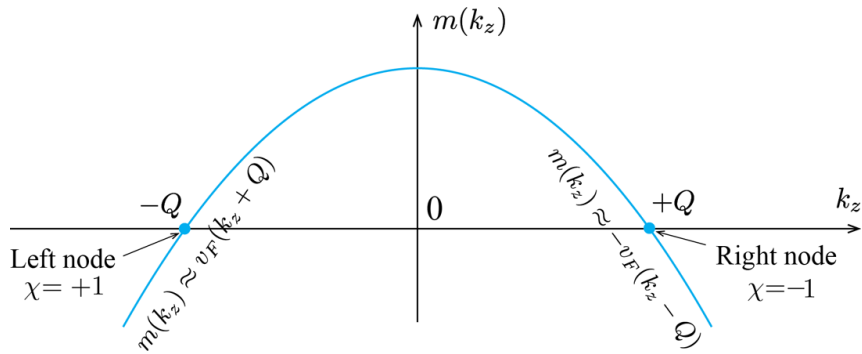

FIG. 1. The function $m\left(k_{z}\right)$ which determines the dispersion of the quasiparticles as a function of the momentum $k_{z}$ for $k_{x}=k_{y}=0$ [cf. Eq. (3.1)].

quasiparticle momentum (hereafter $\hbar=1) ; \hat{\sigma}=\left(\hat{\sigma}_{x}, \hat{\sigma}_{y}, \hat{\sigma}_{z}\right)$ is the pseudospin operator, a degree of freedom equivalent to a spin $1 / 2$; the vector potential $\mathbf{A}=\left(A_{x}, A_{y}, A_{z}\right)$ accounts for the magnetic field; $\mathbf{R}_{i}$ is the location of the $i$ th impurity; $Z_{i} e$ is the charge of the $i$ th impurity; $\varkappa$ is the inverse screening length which is specified below; and $\varepsilon$ is the dielectric constant. For simplicity, we consider only two types of impurities: with $Z_{i}=+1$ (donors) and with $Z_{i}=-1$ (acceptors).

In Eq. (3.1), we also introduced a nonlinear function $m\left(k_{z}\right)$ which may be approximated by the linear dependencies $m\left(k_{z}\right) \approx v_{F}\left(Q \mp k_{z}\right)$ near the Weyl nodes, located at the momenta $\mathbf{k}=(0,0, \pm Q)$, as shown in Fig. 1, where the prefactor $v_{F}$ is assumed, for simplicity, to match the velocity of the quasiparticles along the $x$ and $y$ axes in the absence of the magnetic field. The free quasiparticles, thus, have linear (Weyl) dispersions near the nodes with the velocity $v_{F}$ and with opposite chiralities [52] $\chi= \pm 1$ at the respective nodes. The values of $m\left(k_{z}\right)$ away from the nodes determine the amplitude of quasiparticle tunnelling between the nodes in momentum space. Such tunnelling is usually neglected in studies of transport in WSMs. In this paper, we demonstrate, however, that the internodal tunnelling has a qualitative effect on magnetotransport for certain directions of the magnetic field.

We choose the $x z$ plane to be parallel to the direction of the magnetic field, as well as to the separation between the Weyl nodes in momentum space. In what follows, we use the Landau gauge,

$$
\mathbf{A}=(B y \cos \eta, 0,-B y \sin \eta),
$$

where $\eta$ is the angle between the direction of the magnetic field $\mathbf{B}$ and the $z$ axis, as shown in Fig. 3.

The magnetic field leads to the quantization of the quasiparticle motion in the plane perpendicular to the field in a sufficiently clean system. While we expect our results to hold for arbitrary magnetic fields, we consider, for simplicity, the ultraquantum limit, where only the zeroth Landau level contributes to the transverse motion. This limit corresponds to sufficiently strong magnetic fields,

$$
B>\frac{\mu^{2} c}{2 e^{2} v_{F}^{2}},
$$

where $\mu$ is the chemical potential measured from the energy of the Weyl nodes. We also assume that $Q$ is the largestmomentum scale in the problem and, in particular, exceeds 


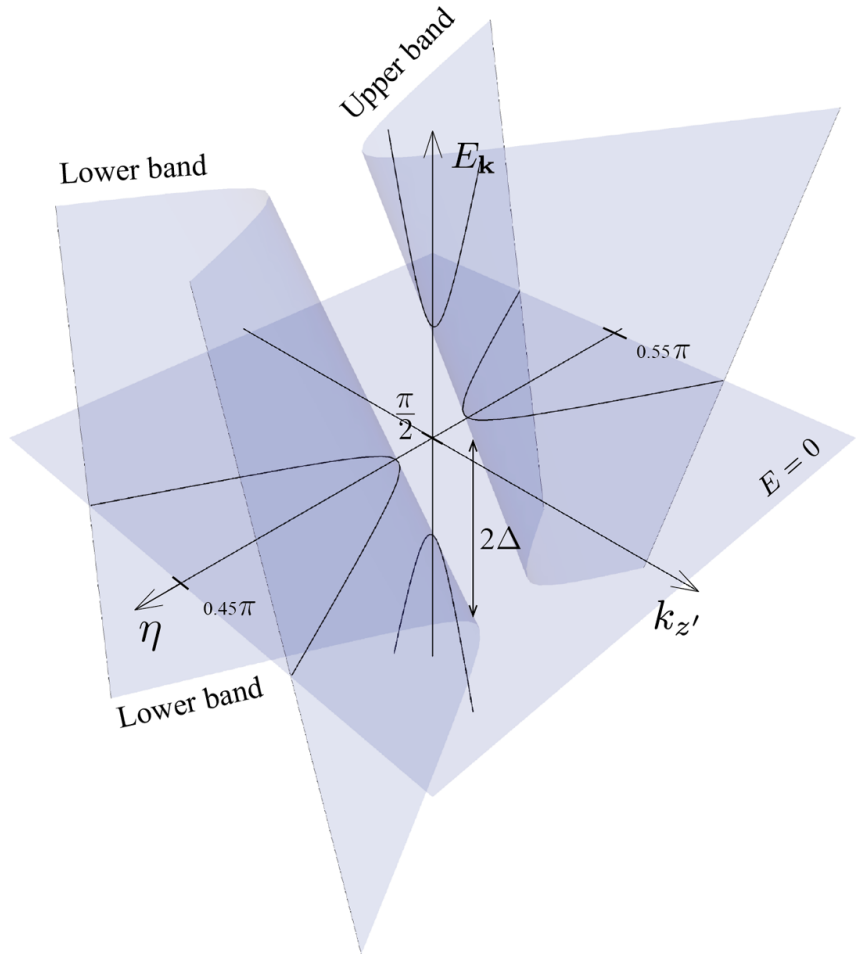

FIG. 2. The dispersion $E_{\mathbf{k}}$ of the quasiparticles in a Weyl semimetal in a magnetic field at the zeroth Landau level as a function of the momentum $k_{z^{\prime}}$ along the magnetic field and the angle $\eta$ between the field and the separation between the nodes for $\eta$ close to $\frac{\pi}{2}$. The dispersion has two bands, the "upper band" and the "lower band." The " $E=0$ " plane corresponds to the energies of the Weyl nodes in an undoped system.

the inverse magnetic length,

$$
\frac{1}{l_{B}}=\sqrt{\frac{|e| B}{c}} .
$$

Impurity potential. The last term in the Hamiltonian (3.1) describes the potential of the screened impurities. The inverse screening radius $\varkappa$ is given, in the Thomas-Fermi approximation and in the ultraquantum limit under consideration (see Appendix A for details) by

$$
\varkappa=\sqrt{\frac{2|e|^{3} B}{\pi \epsilon v_{F} c}} \equiv \sqrt{\frac{2 \alpha}{\pi}} \frac{1}{l_{B}},
$$

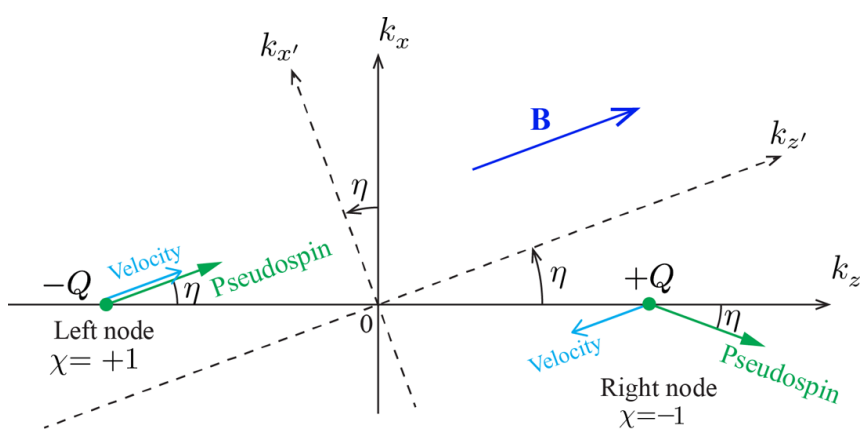

FIG. 3. Weyl nodes and the directions of the magnetic field, quasiparticle velocities, and the pseudospins near the nodes. where $l_{B}$ is the magnetic length, given by Eq. (3.4), and $\alpha=\frac{e^{2}}{v_{F} \epsilon}$ is the "fine-structure constant." Because the dielectric constant $\epsilon$ is large [48-51] in most Weyl and Dirac materials, the fine-structure constant $\alpha$ may be assumed to be small, which justifies using the Thomas-Fermi approximation when describing electrostatic screening in these systems (see Appendix A for a more detailed discussion). A factor of 2 in Eq. (3.5) reflects the number of Weyl nodes in the system.

Donor and acceptor impurities affect the concentration of the quasiparticles and, thus, determine the chemical potential in the system. In an uncompensated system, i.e., at $n_{A} \neq n_{D}$, the chemical potential $\mu$ at realistic dopant concentrations may be considered homogeneous, with the average value significantly exceeding spatial fluctuations [53]. By contrast, in well-compensated systems, with $\left|\frac{n_{A}-n_{D}}{n_{A}+n_{D}}\right| \ll \alpha^{\frac{3}{2}}$, the fluctuations of the screened potential of the impurities are significant and lead to the formation of electron and hole puddles [53,54]. In this paper, we focus on the regime of an uncompensated system, as more experimentally relevant, with a sufficiently homogeneous chemical potential.

Charge neutrality requires that

$$
n_{D}-n_{A}=\frac{B|e| \mu}{2 \pi^{2} v_{F} c}
$$

where $n_{A}$ and $n_{D}$ are the concentrations of the acceptor and donor impurities. The right-hand side of Eq. (3.6) gives the concentration of quasiparticles measured from that in a disorder-free system with $\mu=0$, which is related to the chemical potential $\mu_{0}$ in the absence of the magnetic field as $n_{D}-n_{A}=\frac{\mu_{0}^{3}}{3 \pi^{2} v_{F}^{3}}$. Because the density of states of the charge carriers is affected by the magnetic field and their concentration $n_{D}-n_{A}$ is field independent, the chemical potential $\mu$ depends on the magnetic field. According to Eq. (3.6),

$$
\mu(B)=\frac{2 c \mu_{0}^{3}}{3 B|e| v_{F}^{2}} .
$$

\section{QUASIPARTICLE DISPERSION IN A DISORDER-FREE SEMIMETAL}

In this section, we analyze the dispersion of the quasiparticles at the zeroth Landau level in a disorder-free system. Figure 2 shows the dispersion, obtained by computing numerically the eigenenergies of the Hamiltonian (3.1) in the absence of impurities, as a function of the momentum $k_{z^{\prime}}$ along the magnetic field and the angle $\eta$ between the field and the separation between the nodes for $\eta$ close to $\pi / 2$.

The dispersion consists of two bands (the "upper band" and "lower band" in Fig. 2) separated by a gap $2 \Delta$, which is determined by the internodal tunnelling of the quasiparticles. For $\eta=\pi / 2$, the dispersion is particle-hole symmetric, i.e., the two branches of the dispersion are symmetric with respect to $E=0$. For angles $\eta$ deviating from $\pi / 2$, the energies of both branches are shifted. At large momenta, $\left|k_{z^{\prime}}\right| \gg \Delta / v_{F}$, the dispersion is linear as a function of momentum. In what follows, we describe the dispersion analytically. 


\section{A. Quasiparticle dispersion and wave functions for decoupled $\operatorname{nodes}(\Delta=0)$}

First, we describe the dispersion and the wave functions of low-energy quasiparticles neglecting the internodal tunnelling. This corresponds to either a negligible gap $2 \Delta$, e.g., due to the magnetic field being small, or to angles $\eta$ sufficiently away from $\pi / 2$, where the dispersion of low-energy quasiparticles (near the energy $E=0$ ) is described by the linear parts of the dispersion branches, independent of the quantity $\Delta$.

We linearize the function $m\left(k_{z}\right)$ in the Hamiltonian (3.1) near the node of chirality $\chi$ (cf. Fig. 1),

$$
m\left(k_{z}\right) \approx v_{F}\left(Q+\chi k_{z}\right),
$$

and rotate the coordinate frame by the angle $\eta$ about the $y$ axis, as shown in Fig. 3. The $z^{\prime}$ axis of the rotated coordinate frame $x^{\prime} y z^{\prime}$ is parallel to the direction of the magnetic field $\mathbf{B}$ and the components of the quasiparticle momenta in the $x^{\prime} z^{\prime}$ plane are given by

$$
\begin{aligned}
& k_{x^{\prime}}=k_{x} \cos \eta-k_{z} \sin \eta, \\
& k_{z^{\prime}}=k_{x} \sin \eta+k_{z} \cos \eta .
\end{aligned}
$$

It is convenient to also rotate the pseudospin basis at each node of chirality $\chi$ by the angle $\chi \eta$ about the $y$ axis in the pseudospin space, which corresponds to the transformation of the Hamiltonian given by

$$
\hat{H} \rightarrow \hat{H}^{\prime}=e^{\frac{i x \lambda \hat{\theta}_{y}}{2}} \hat{H} e^{-\frac{i \chi \chi \hat{\sigma}_{y}}{2}} .
$$

The Hamiltonian

$$
\begin{aligned}
\hat{H}_{0}^{\prime}= & v_{F}\left(Q \cos \eta+\chi k_{z^{\prime}}\right) \hat{\sigma}_{z} \\
& +v_{F}\left(k_{x^{\prime}}-\chi Q \sin \eta-\frac{e}{c} B y\right)-i v_{F} \partial_{y}
\end{aligned}
$$

of free quasiparticles near the nodes in the rotated pseudospin basis may be rewritten conveniently, by introducing the annihilation

$$
\hat{a}=\frac{1}{l_{B} \sqrt{2}}\left(\partial_{y}+k_{x^{\prime}}-\chi Q \sin \eta-\frac{e}{c} B y\right),
$$

and creation $\hat{a}^{\dagger}$ ladder operators of a harmonic oscillator, in the form

$$
\hat{H}_{0}^{\prime}=v_{F}\left(Q \cos \eta+\chi k_{z^{\prime}}\right) \hat{\sigma}_{z}+\frac{1}{\sqrt{2}} \frac{v_{F}}{l_{B}} \hat{\sigma}^{+} \hat{a}^{\dagger}+\frac{1}{\sqrt{2}} \frac{v_{F}}{l_{B}} \hat{\sigma}^{-} \hat{a},
$$

where $\hat{\sigma}^{ \pm}=\hat{\sigma}_{x} \pm i \hat{\sigma}_{y}$.

The Hamiltonian (4.7) with the ladder operator (4.6) describes quasiparticles which propagate parallel (or antiparallel) to the $z^{\prime}$ axis, i.e., along the direction of the magnetic field $\mathbf{B}$, and whose motion in the transverse direction is quantized. The eigenstates of this Hamiltonian are parametrized by the component $k_{z^{\prime}}$ of momentum parallel to the magnetic field, the number $n$ of the Landau level of the transverse motion, and the transverse component $k_{x^{\prime}}$ of momentum. As discussed in Secs. II and III, we focus on the case of sufficiently strong magnetic fields, at which only the zeroth Landau levels contribute to transport.
The respective eigenstates of the Hamiltonian (4.7) are given by

$$
\left|\psi_{\chi}\right\rangle=|\uparrow\rangle_{\chi} \otimes|0\rangle_{\mathrm{LL}}\left(k_{x^{\prime}}\right) \otimes\left|k_{z^{\prime}}\right\rangle,
$$

where $|\uparrow\rangle_{\chi}$ is the state of the pseudospin directed along the $z$ axis in the pseudospin space [where the pseudospin basis depends on the chirality of the node $\chi$; cf. (4.4)], $|0\rangle_{\mathrm{LL}}$ is the eigenstate of the operator $\hat{a}$ corresponding to the zeroth Landau level and parametrized by the momentum component $k_{x^{\prime}}$, and $\left|k_{z^{\prime}}\right\rangle$ describes a plane-wave wave function of a particle with momentum $k_{z^{\prime}}$ along the $z^{\prime}$ axis. In the coordinate representation and the pseudospin basis of the Hamiltonian (3.1), the eigenstate is given by

$$
\begin{aligned}
\psi_{\chi \mathbf{k}}(\mathbf{r})= & e^{-\frac{\left(c k_{x^{\prime}}-c \chi Q \sin \eta-e B y\right)^{2}}{2|e| B}+i k_{z^{\prime}} z^{\prime}+i k_{x^{\prime}} x^{\prime}} \\
& \times\left(\frac{|e| B}{\pi c L_{z^{\prime}}^{2} L_{x^{\prime}}^{2}}\right)^{\frac{1}{4}}\left(\begin{array}{c}
\cos \frac{\eta}{2} \\
\chi \sin \frac{\eta}{2}
\end{array}\right),
\end{aligned}
$$

where we assumed that the system has finite sizes $L_{z^{\prime}}$ and $L_{x^{\prime}}$ along the $z^{\prime}$ and $x^{\prime}$ axes; $\mathbf{k}$ is the momentum in the $x z$ plane (that matches the $x^{\prime} z^{\prime}$ plane). The energy of this eigenstate is determined by the first term in the effective Hamiltonian (4.7) and is given by

$$
E_{\chi \mathbf{k}}=v_{F}\left(Q \cos \eta+\chi k_{z^{\prime}}\right) .
$$

The pseudospins of the low-energy quasiparticles under consideration have different directions, shown in Fig. 3, at nodes of different chiralities $\chi$. As follows from Eq. (4.10), the quasiparticle velocities $\mathbf{v}_{\chi \mathbf{k}}=\frac{\partial E_{\chi \mathbf{k}}}{\partial \mathbf{k}}$ at the nodes with $\chi=$ +1 and $\chi=-1$ are, respectively, parallel and antiparallel to the magnetic field, as shown in Fig. 3.

\section{Generic wave function near a node}

Although the Hamiltonian lacks translational invariance in the presence of the magnetic field, a generic wave function, considered as a function of the momentum $\mathbf{k}$ in the $x z$ plane and the coordinate $y$, may still be attributed to a particular node, so long as its generalized momentum $\mathbf{K}=\mathbf{k}-\frac{e}{c} \mathbf{A}$ is close to the momentum of this node in the absence of the magnetic field, where $\mathbf{A}(y)$ is the vector potential given by Eq. (3.2).

Indeed, this condition is equivalent to (1) the component $k_{z^{\prime}}$ of the momentum being close to $-\chi Q \cos \eta$ and (2) the argument $k_{z}-\frac{e}{c} A_{z}$ of the function $m$ in the Hamiltonian (3.1) being close to $-\chi Q$. The first condition guarantees that the wave function is a superposition of eigenstates with low energies (measured from the energy of a node), while the second condition allows us to linearize the function $m\left(k_{z}\right)$ according to Eq. (4.1).

\section{B. Internodal tunnelling}

The Hamiltonian (3.1) describes quasiparticles with a finite bandwidth along the $z$ axis, which allows for quasiparticle tunnelling from one node to the other. Although this tunnelling is exponentially suppressed by this bandwidth, which exceeds all the other energy scales in the problem, it leads to the opening of the gap $2 \Delta$, shown in Fig. 2, in the quasiparticle dispersion. In this section, we consider analytically the effect 
of such internodal tunnelling in momentum space on the quasiparticle dispersion and the opening of the gap.

The tunnelling leads to the hybridization of low-energy quasiparticle states near different nodes. The generic wave function $\psi(\mathbf{r})$ of a quasiparticle with momentum components $k_{x}$ and $k_{z}$ in the $x z$ plane and with a sufficiently low energy (measured from the energy of the nodes) may be approximated as a superposition of the wave functions (4.9) belonging to the nodes with $\chi=+1$ and $\chi=-1$.

\section{Conditions for strong internodal hybridization}

Quasiparticle states at different nodes with the same momentum $k_{z^{\prime}}$ are strongly hybridized by the internodal tunnelling if these states have the same energies in the absence of the tunnelling. Two quasiparticles states near the nodes with $\chi=+1$ and $\chi=-1$ have close energies (4.10) if the momentum $k_{z^{\prime}}$ is sufficiently small. Because the energies under consideration are also small, this leads to the requirement of the smallness of the momentum scale $Q \cos \eta$ in comparison with the separation $Q$ between the nodes in momentum space and the characteristic momentum scale associated with the internodal tunnelling discussed below.

Therefore, the states at different nodes may be strongly hybridized only for angles $\eta$ sufficiently close to $\pi / 2$,

$$
\left|\eta-\frac{\pi}{2}\right| \ll \frac{\Delta}{v_{F} Q},
$$

where $\Delta$ is the characteristic coupling energy between the states with $\chi=+1$ and $\chi=-1$.

Strong hybridization requires not only that the angle $\eta$ be close to $\pi / 2$, but also that the energies of the hybridized states be close to each other. At $\eta=\pi / 2$, the energies of the states at nodes $\chi=+1$ and $\chi=-1$ are given by $E_{+1 \mathbf{k}}=$ $-E_{-1 \mathbf{k}}=v_{F} k_{z^{\prime}}=v_{F} k_{x}$, according to Eq. (4.10). Therefore, strong hybridization of states between nodes also requires that

$$
v_{F}\left|k_{x}\right| \ll \Delta,
$$

in addition to the condition (4.11).

\section{Internodal coupling}

At angles $\eta$ very close to $\pi / 2$, i.e., for magnetic fields almost perpendicular to the separation between the nodes, the nodes are strongly coupled by the tunnelling, and the effective Hamiltonian of low-energy quasiparticles is given by

$$
\begin{aligned}
\hat{\mathcal{H}}_{\text {internodal }}(\mathbf{k})= & v_{F}\left(Q \cos \eta+k_{z^{\prime}}\right)\left|\psi_{+1 \mathbf{k}}\right\rangle\left\langle\psi_{+1 \mathbf{k}}\right| \\
& +v_{F}\left(Q \cos \eta-k_{z^{\prime}}\right)\left|\psi_{-1 \mathbf{k}}\right\rangle\left\langle\psi_{-1 \mathbf{k}}\right| \\
& +\Delta\left(\left|\psi_{+1 \mathbf{k}}\right\rangle\left\langle\psi_{-1 \mathbf{k}}|+| \psi_{-1 \mathbf{k}}\right\rangle\left\langle\psi_{+1 \mathbf{k}}\right|\right),
\end{aligned}
$$

where $\mathbf{k}$ is the momentum in the $x z$ plane, the states $\left|\psi_{\chi \mathbf{k}}\right\rangle$ are given by Eqs. (4.8) and (4.9), the first two lines describe the dispersion near decoupled nodes, and $\Delta$ determines the strength of the coupling. At $\eta=\frac{\pi}{2}$, the quantity $2 \Delta$ gives the gap in the low-energy quasiparticle dispersion,

$$
E_{\mathbf{k}}= \pm\left(v_{F}^{2} k_{x}^{2}+\Delta^{2}\right)^{\frac{1}{2}}
$$

shown in Fig. 2 (in the $\eta=\pi / 2$ plane).
The amplitude of the tunnelling between the two nodes may be computed (see Appendix C) by matching the wave functions of the quasiparticles near the nodes with exponentially small "tails" between the nodes obtained in the quasiclassical approximation. Recently, the internodal coupling for a particular form of the function $m\left(k_{z}\right)$ [cf. Eq. (3.1)] has been computed in Ref. [39]. In Appendix C, we generalize this calculation to the case of an arbitrary function $m\left(k_{z}\right)$, with the result

$$
\Delta=v_{F} \sqrt{\frac{|e| B}{\pi c}} \exp \left[-\frac{c}{|e| B v_{F}} \int_{-Q}^{Q} m\left(k_{z}\right) d k_{z}\right] .
$$

Equation (4.15) is valid for small values of the exponential, which reflects strong suppression of the quasiparticle wave functions away from the nodes. When deriving Eq. (4.15), we also use the smallness of the inverse magnetic length (3.4) compared to the separation $2 Q$ between the nodes.

\section{MAGNETOCONDUCTIVITY AWAY FROM $\eta=\pi / 2$}

In this section, we consider magnetotransport for angles $\eta$ away from $\pi / 2$, at which the internodal tunnelling may be neglected and quasiparticles at different nodes are effectively decoupled. Because intranodal scattering processes do not change the velocities of the quasiparticles, shown in Fig. 3, the resistivity of the system in this regime is determined by the processes of internodal scattering.

As discussed in Sec. IV, the component $\mathbf{k}$ of momentum in the $x z$ plane is a good quantum number in a disorder-free system for the choice of the gauge of the vector potential given by Eq. (3.2). Internodal scattering occurs between quasiparticle states $\psi_{\chi \mathbf{p}}$ and $\psi_{-\chi \mathbf{k}}$ with momenta $\mathbf{p}$ and $\mathbf{k}$ separated by a vector of approximate length $2 Q$. While the difference between the momenta of quasiparticles near different nodes is evident in the absence of magnetic field, it requires a clarification in the presence of a magnetic field due to the lack of translational invariance of the Hamiltonian.

Indeed, the change of the generalized momentum $\mathbf{k}-$ $\frac{e}{c} \mathbf{A}(y)$ of a scattered quasiparticle is close to $2 Q$. This leads to the difference between the incoming and outgoing momenta $\mathbf{p}$ and $\mathbf{k}$ being close to $2 Q$ if the random potential is sufficiently smooth. The potential of the screened impurities is smooth at length scales that are shorter than the screening length, $\varkappa^{-1} \sim l_{B} / \alpha^{\frac{1}{2}}$. Therefore, the difference between the momenta $\mathbf{p}$ and $\mathbf{k}$ is close to $2 Q$ as long as momentum scales of the order of $\alpha^{\frac{1}{2}} / l_{B} \ll Q$ are neglected. This conclusion may also be obtained by explicitly evaluating the matrix elements of scattering of the states of the form (4.9) on the potential of screened impurities (see Appendix D for details).

The scattering rate of the state $\psi_{\chi \mathbf{p}}$ at a node with chirality $\chi$ to the other node, with chirality $-\chi$, is given by

$$
\frac{1}{\tau_{\chi \mathbf{p}}}=2 \pi \int\left\langle\left|\left\langle\psi_{\chi \mathbf{p}}|U| \psi_{-\chi \mathbf{k}}\right\rangle\right|^{2}\right\rangle_{\mathrm{dis}} \delta\left(E_{\chi \mathbf{p}}-E_{-\chi \mathbf{k}}\right) \frac{S_{x z} d \mathbf{k}}{(2 \pi)^{2}},
$$

where $U$ is the potential [55] of randomly located screened impurities; $\langle\ldots\rangle_{\text {dis }}$ is our convention for the averaging over the locations of the impurities; $E_{\chi \mathbf{p}}$ is the energy of the state with momentum $\mathbf{p}$ near node $\chi$; and $S_{x z}$ is the cross-sectional 
area of the system in the $x z$ plane (which, for simplicity, is assumed to be constant along the $y$ axis).

The potential $U(\mathbf{r})$ is given by the last term in the Hamiltonian (3.1) and is a sum of the potentials $u_{i}(\mathbf{r})=Z_{i} \frac{e^{2}}{\epsilon} \frac{e^{-\varkappa \mid r}-\mathbf{R}_{i} \mid}{\left|\mathbf{r}-\mathbf{R}_{i}\right|}$ of individual impurities at locations $\mathbf{R}_{i}$. In this paper, we neglect single-particle interference effects related to scattering off multiple impurities [56,57], assuming that the concentration of impurities is small.

Under this approximation, we may make the replacement

$$
\begin{aligned}
& \left\langle\left|\left\langle\psi_{\chi \mathbf{p}}|U(\mathbf{r})| \psi_{-\chi \mathbf{k}}\right\rangle\right|^{2}\right\rangle_{\mathrm{dis}} \\
& \quad \rightarrow \sum_{i} \frac{1}{V} \int d \mathbf{R}_{i}\left|\left\langle\psi_{\chi \mathbf{p}}\left|u\left(\mathbf{r}-\mathbf{R}_{i}\right)\right| \psi_{-\chi \mathbf{k}}\right\rangle\right|^{2}
\end{aligned}
$$

in the expression (5.1) for the scattering time. Using Eq. (5.2) and evaluating the integral in Eq. (5.1) (see Appendix D for the details), we arrive at the internodal scattering rate,

$$
\frac{1}{\tau} \approx \frac{2 \pi n|e| B}{v_{F} c}\left(\frac{e^{2} \cos \eta}{2 \varepsilon Q^{2}}\right)^{2},
$$

which is independent of the momentum of the scattered quasiparticle, where $n=N / V$ is the concentration of the impurities in the system.

The dependence $\propto \cos ^{2} \eta$ of the scattering rate on the angle $\eta$ reflects the projection of the pseudospins of quasiparticles at different nodes, shown in Fig. 3, onto each other. Equation (5.3) suggests that the internodal scattering vanishes at $\eta=$ $\pi / 2$ as the pseudospins at different nodes are opposite to each other if the magnetic field is perpendicular to the line separating the nodes. We emphasize, however, that the result (5.3) for the scattering rate applies at angles $\eta$ which are not very close to $\pi / 2$, at which the hybridization between quasiparticle states at different nodes may be neglected.

Because the quasiparticles in an impurity-free system move parallel to the magnetic field, the longitudinal conductivity (along the magnetic field) of a weakly disordered system significantly exceeds its transverse conductivity. When computing the longitudinal conductivity, on which we focus in this paper, transport may, therefore, be considered to be one dimensional, with $N_{\perp}=\frac{|e| B}{2 \pi c}$ transverse channels per crosssectional unit area.

Since conduction comes only from low-energy quasiparticles, with energies significantly exceeded by the bandwidth, their distribution function $f\left(k_{x^{\prime}}, k_{z^{\prime}} \mathbf{r}\right)$ in the space of the momentum $\mathbf{k}=\left(k_{x^{\prime}}, k_{z^{\prime}}\right)$ in the $x z$ plane and three-dimensional (3D) coordinates $\mathbf{r}$ is peaked sharply near the surfaces $k_{z^{\prime}}=$ $-\chi Q \cos \eta ; k_{x^{\prime}}=\chi Q \sin \eta+\frac{e B}{c} y$, corresponding to node $\chi$, according to the discussion in Sec. IV A. Because the dynamics of the quasiparticles is effectively one dimensional and confined to the zeroth Landau level in the transverse direction, it is convenient to introduce the distribution function $F\left(k_{z^{\prime}}, \mathbf{r}\right)=\int_{\chi} \frac{d k_{x^{\prime}}}{2 \pi} f\left(k_{z^{\prime}}, k_{x^{\prime}} \mathbf{r}\right)$ of the longitudinal momentum $k_{z^{\prime}}$ and coordinate near node $\chi$, where the integration with respect to the momentum $k_{x^{\prime}}$ is carried out near that node.

The dynamics of this distribution function is governed by the kinetic equation

$$
\begin{aligned}
& \frac{\partial F_{\chi}\left(k_{z^{\prime}}, \mathbf{r}\right)}{\partial t}+\chi v_{F} \frac{\partial F_{\chi}\left(k_{z^{\prime}}, \mathbf{r}\right)}{\partial z^{\prime}}+e E_{z^{\prime}} \frac{\partial F_{\chi}\left(k_{z^{\prime}}, \mathbf{r}\right)}{\partial k_{z^{\prime}}} \\
& =\frac{F_{-\chi}\left(-k_{z^{\prime}}, \mathbf{r}\right)-F_{\chi}\left(k_{z^{\prime}}, \mathbf{r}\right)}{\tau}
\end{aligned}
$$

for momenta $k_{z^{\prime}}$ close to the value $k_{z^{\prime}} \approx \pm Q \cos \eta$ near a node of chirality $\chi$. Equation (5.4) reflects that elastic internodal scattering leads to the scattering of states with momentum $k_{z^{\prime}}$ along the $z^{\prime}$ axis into the states with momentum $-k_{z^{\prime}}$, as follows from the conservation of energy (4.10).

The generic stationary solution of the kinetic equation (5.4) in a homogeneous system in the presence of a small electric field $E_{z^{\prime}}$ is given by

$$
F_{\chi}\left(k_{z^{\prime}}+e E_{z^{\prime}} \tau\right)=F_{-\chi}\left(-k_{z^{\prime}}\right),
$$

which leads to the current in the $z^{\prime}$ direction,

$$
j=e N_{\perp} v_{F} \int\left[F_{+1}\left(k_{z^{\prime}}\right)-F_{-1}\left(k_{z^{\prime}}\right)\right] \frac{d k_{z^{\prime}}}{2 \pi}=\frac{|e|^{3} v_{F} B \tau}{(2 \pi)^{2} c} E_{z^{\prime}} .
$$

Utilizing Eqs. (5.6) and (5.3) and recovering the Planck's constant $\hbar$, we arrive at the conductivity

$$
\sigma_{\text {inter }}=\frac{v_{F}^{2} Q^{4} \hbar \varepsilon^{2}}{2 \pi^{3} n e^{2}} \frac{1}{\cos ^{2} \eta},
$$

which describes the first contribution to the resistivity in Eq. (2.1) with $\rho_{\text {inter }}$ given by (2.4).

As discussed in Sec. II, the conductivity (5.7) is weakly dependent on the magnitude of the magnetic field, but exhibits strong anisotropy, i.e., strong dependence on the orientation of magnetic field with respect to the separation between the Weyl nodes. A strong dependence of the conductivity on the direction of the magnetic field has also been noted in Ref. [58]; however, with a different dependence on the direction. We believe that the difference comes from assuming in Ref. [58], without a derivation for a particular model, a certain structure of the kinetic energy and the disorder potential in the space of the nodal spin. We also emphasize the absence of the dependence of the conductivity (5.7) on the amplitude of the magnetic field.

\section{MAGNETOCONDUCTIVITY AT ANGLES $\eta$ CLOSE TO $\pi / 2$}

In this section, we consider magnetotransport at angles $\eta$ close to $\pi / 2$, i.e., for the magnetic field $\mathbf{B}$ parallel to the $x$ axis. In this case, quasiparticle states at different nodes are coupled strongly by the internodal tunnelling. As discussed in Sec. V, the internodal coupling essentially determines the resistivity in this regime, as the conductivity (5.7) diverges at $\eta=\pi / 2$.

We assume here that the chemical potential $\mu$ (measured from the energy of the Weyl nodes in the absence of the magnetic field) is greater than the internodal coupling $\Delta$. Indeed, the chemical potential $\mu$ is of the order of several dozen meV for typical doping levels in WSMs (see, e.g., Refs. $[54,59,60])$, whereas the coupling is of the order of several meV for fields $B \sim 10 T$ and decreases exponentially for smaller fields.

In this approximation, the quasiparticle states in the absence of disorder may be represented in the form

$$
\left|\phi_{\chi \mathbf{k}}\right\rangle \approx\left|\psi_{\chi \mathbf{k}}\right\rangle+\frac{\chi \Delta}{2 v_{F} k_{x}}\left|\psi_{-\chi \mathbf{k}}\right\rangle,
$$


as follows from the effective Hamiltonian (4.13) of the quasiparticles at $\eta=\pi / 2$. The second term in Eq. (6.1) is small due to the smallness of the parameter $\Delta /\left(k_{x} v_{F}\right) \ll 1$, and accounts for the hybridization between the states $\left|\psi_{+1 \mathbf{k}}\right\rangle$ and $\left|\psi_{-1 \mathbf{k}}\right\rangle$ due to internodal tunnelling, which is essential for the conductivity being finite at $\eta=\pi / 2$.

Quasiparticles with the wave functions $\left|\phi_{+1 \mathbf{k}}\right\rangle$ and $\left|\phi_{-1 \mathbf{k}}\right\rangle$ move with the velocity $v_{F}$ (up to small corrections of the order of $\left.\Delta^{2} / \mu^{2}\right)$, respectively, parallel and antiparallel to the $x$ axis. The resistivity of the system is, thus, determined by the processes of scattering between the states $\left|\phi_{\chi \mathbf{k}}\right\rangle$ with different $\chi$.

The respective "internodal" scattering rate is given by Eq. (5.1) with the replacement $\left|\psi_{\chi \mathbf{k}}\right\rangle \rightarrow\left|\phi_{\chi \mathbf{k}}\right\rangle$. Utilising Eqs. (5.1), (5.2), (6.1), and (4.10), we obtain the scattering rate (see Appendix E for details),

$$
\begin{aligned}
\frac{1}{\tau} \approx & \frac{2 \pi n v_{F} e^{4}}{\varepsilon^{2}\left[4 \mu^{2}(B)+\varkappa^{2}(B) v_{F}^{2}\right]} \frac{\Delta^{2}}{\mu^{2}(B)} \\
& \times\left[1+I\left(l_{B} Q, l_{B} \sqrt{4 \mu^{2}(B)+\varkappa^{2} v_{F}^{2}} / v_{F}\right)\right],
\end{aligned}
$$

where the function $I(s, t)$ is given by

$$
I(s, t)=\frac{t^{2}}{\pi} \int \frac{\cos (2 s y) e^{-\frac{z^{2}+y^{2}}{2}}}{\left(y^{2}+z^{2}+t^{2}\right)^{2}} d z d y,
$$

and the chemical potential $\mu(B)$ is given by Eq. (3.7). Depending on the values of the magnetic field and the chemical potential, the value of the function $I\left(l_{B} Q, l_{B} \sqrt{4 \mu^{2}(B)+\varkappa^{2} v_{F}^{2}} / v_{F}\right)$ may vary from zero, in the limit $Q l_{B} \max \left(\sqrt{\frac{2 \alpha}{\pi}}, \frac{2 \mu l_{B}}{v_{F}}\right) \gg 1$, to unity, in the limit $Q l_{B} \max \left(\sqrt{\frac{2 \alpha}{\pi}}, \frac{2 \mu l_{B}}{v_{F}}\right) \ll 1$ (see Appendix E for details).

For the experimentally relevant case of chemical potentials $|\mu(B)| \gg \Delta$ exceeding the gap $\Delta$ in quasiparticle dispersion, the quasiparticles move with velocities $v_{F}$ and $-v_{F}$ along the $z^{\prime}$ axis and the conductivity of the system is given by the same expression,

$$
\sigma=\frac{|e|^{3} v_{F} B}{(2 \pi)^{2} c} \tau,
$$

as in Sec. V [cf. Eq. (5.6)]. Equations (6.2) and (6.4) give the longitudinal conductivity of a WSM in a magnetic field perpendicular to the line connecting the nodes in the form

$$
\sigma=\frac{\varepsilon^{2} B\left(\mu^{2}+\varkappa^{2} v_{F}^{2}\right)}{(2 \pi)^{3} n|e| c(1+I)} \frac{\mu^{2}}{\Delta^{2}},
$$

where $I=I\left(l_{B} Q, l_{B} \sqrt{4 \mu^{2}(B)+\varkappa^{2} v_{F}^{2}} / v_{F}\right)$ and $\Delta$ is the gap in the dispersion of the quasiparticles at the zeroth Landau level given by Eq. (4.15).

In what immediately follows, we provide the results for conductivity in the two limiting cases: $\mu \gg \varkappa v_{F}$ and $\mu \ll$ $\varkappa v_{F}$. According to Eqs. (3.5) and (3.7), this corresponds, respectively, to the magnetic fields $B \ll B_{\mu}$ and $B \gg B_{\mu}$, where the characteristic field $B_{\mu}$ is given by Eq. (2.6) and is of the order of $0.1 T$ for typical Weyl semimetals. For $B \ll B_{\mu}$,
Eq. (6.4) gives

$$
\sigma=\frac{2 \varepsilon^{2} c^{4} \mu_{0}^{12}}{81 \pi^{2} n e^{6} v_{F}^{10}(1+I)} \frac{1}{B^{4}} \exp \left[\frac{2 c}{|e| B v_{F}} \int_{-Q}^{Q} m\left(k_{z}\right) d k_{z}\right] ;
$$

for $B \gg B_{\mu}$

$$
\sigma=\frac{\varepsilon c \mu_{0}^{6}}{9 \pi^{3} n|e| v_{F}^{5}(1+I)} \frac{1}{B} \exp \left[\frac{2 c}{|e| B v_{F}} \int_{-Q}^{Q} m\left(k_{z}\right) d k_{z}\right] .
$$

Combining Eqs. (6.6) and (6.7) and recovering the Planck's constant $\hbar$ gives the interpolation formula (2.5).

\section{SUMMARY AND OUTLOOK}

In summary, we studied transport in a Weyl semimetal in a strong magnetic field in the presence of Coulomb impurities and focusing on the case of a two-node Weyl semimetal. The resistivity in the direction of the magnetic field exhibits a strong dependence, $\propto \cos \eta^{2}+C$, on the angle $\eta$ between the direction of the magnetic field and the separation between the Weyl nodes in momentum space, where $C \ll 1$ is a small constant determined by the hybridization of electron states between the nodes.

The strong directional dependence $\propto \cos ^{2} \eta$ of the resistivity (away from the selected directions corresponding to $\eta=$ $\pi / 2$ ) of the system along the magnetic field may be directly compared with the results of experiments on magnetotransport in Weyl semimetals and used, in particular, to identify new Weyl semimetals. At the same time, the resistivity $\rho(B)$ along the directions $\eta=\pi / 2$ allows one to directly probe the internodal coupling $\Delta$, caused by the internodal tunnelling, which may affect phenomena involving internodal dynamics in Weyl semimetals, such as hydrodynamic electron flows [29-36] or quantum oscillations of the resistance [26,27], as well as valleytronic applications, if the magnetic field is perpendicular to the separation between the Weyl nodes.

We expect similarly anisotropic behavior of the longitudinal resistivity in nonuniformly strained Weyl semimetals in the absence of the magnetic field, as elastic strain is known to generate gauge fields similar to those of the magnetic fields having different signs for Weyl fermions of different chiralities [61-63]. Internodal electron dynamics in a strained Weyl semimetal, however, requires a separate analysis, which we leave for future studies.

\section{ACKNOWLEDGMENTS}

We are grateful to Ya. I. Rodionov for useful discussions and previous collaboration on related topics. Our work has been supported financially by the Hellman Foundation and the Faculty Research Grant awarded by the Committee on Research from the University of California Santa Cruz (S.V.S.) and by the Basic Research Programme of the HSE University and the RAS programme "Low Temperature Physics" (K.S.T.). 


\section{APPENDIX A: SCREENING OF IMPURITIES IN A STRONG MAGNETIC FIELD}

In this section, we discuss the screening of a charged impurity in a Weyl semimetal in the presence of a magnetic field. The electrostatic potential $\phi$ around an impurity of charge $Z e$ located at $\mathbf{r}=0$ is given by

$$
\varepsilon \Delta \phi(\mathbf{r})=4 \pi Z e \delta(\mathbf{r})+4 \pi e n(\mathbf{r}),
$$

where $n(\mathbf{r})$ is the change of the density of the electrons due to exposing the system to the potential $\phi$ at location $\mathbf{r}$. In the Thomas-Fermi approximation [56], the response of the electron density $n(\mathbf{r})$ to the potential $\phi(\mathbf{r})$ is local, which gives

$$
\begin{aligned}
n(\mathbf{r}) & =N_{\text {nodes }} \frac{|e| B}{2 \pi c} \int \frac{d k_{z}}{2 \pi}\left\{n_{F}\left[v_{F} k_{z}-e \phi(\mathbf{r})\right]-n_{F}\left(v_{F} k_{z}\right)\right\} \\
& =-N_{\text {nodes }} \frac{e^{2} B \phi}{\left(2 \pi^{2}\right) c v_{F}},
\end{aligned}
$$

where $\frac{|e| B}{2 \pi c}$ accounts for the degeneracy of the Landau level; $v_{F} k_{z}$ is the dispersion of the quasiparticle along the magnetic field; $N_{\text {nodes }}$ is the number of Weyl nodes in the system (in the rest of the paper, $N_{\text {nodes }}=2$ ), and $n_{F}(\varepsilon)$ is the Fermi-Dirac distribution function.

Equations (A1) and (A2) describe linear screening with the radius $\varkappa^{-1}$, which for $N_{\text {nodes }}=2$ is given by Eq. (3.5). We note that the impurity screening may be neglected when considering the direct internodal scattering because the change of momentum for such scattering processes is close to $2 Q$ and significantly exceeds the screening constant $\varkappa$. Therefore, our results for the conductivity obtained in Sec. V, e.g., Eqs. (2.4) and (5.7), are independent of the model of screening.

The screening and the details of the screened potential may be important, however, when considering the transport of quasiparticle states that are strongly hybridized between the nodes. The respective contribution to the resistivity, considered in Sec. VI, is determined by small momentum scattering of the order of $\min \left(|\mu| / v_{F}, \varkappa\right)$, where $\mu$ is the chemical potential in the system.

Thomas-Fermi approximation, which we used to obtain Eq. (A2) and the form of the screened potential given by the last term of the Hamiltonian (3.1), is justified provided the screening length $\varkappa^{-1}$ exceeds the characteristic lengths of the wave functions of the electrons which provide screening: the magnetic length $l_{B}$ and the wavelength $v_{F} / \mu$ corresponding to the motion along the field. The condition $\varkappa^{-1} \gg l_{B}$ is fulfilled in typical Weyl semimetals due to the smallness of the "fine-structure constant" discussed in Sec. III. According to Eqs. (3.5) and (3.7), the condition

$$
\varkappa^{-1} \gg v_{F} / \mu
$$

is fulfilled as long as the magnetic field $B$ is significantly smaller than the characteristic value given by Eq. (2.6). The Thomas-Fermi approximation, used here, breaks down if the magnetic field exceeds this characteristic value, which may affect the preexponential in Eq. (6.7) and in Eq. (2.5) in the limit $B \gg B_{\mu}$; however, the results should still be expected to hold qualitatively.

\section{APPENDIX B: LANDAU LEVELS OF ELECTRONS NEAR DECOUPLED NODES}

In this section, we present a detailed derivation of the wave functions of the quasiparticle near each Weyl node neglecting the internodal tunnelling. The Hamiltonian of a disorderfree Weyl semimetal with two nodes and the quasiparticle dispersion linearized near the nodes is given by

$$
\begin{aligned}
\hat{H}_{0}= & v_{F}\left(k_{x}-\frac{e}{c} B y \cos \eta\right) \hat{\sigma}_{x}-i v_{F} \hat{\sigma}_{y} \partial_{y} \\
& +v_{F}\left(Q+\chi k_{z}+\chi \frac{e}{c} B y \sin \eta\right) \hat{\sigma}_{z},
\end{aligned}
$$

where the momenta $k_{x}$ and $k_{z}$ along the $x$ and $z$ axes are good quantum numbers. The eigenfunction $\psi_{k_{x} k_{z} \chi}$ of a quasiparticle at node $\chi$ and its eigenstate $E_{\chi}^{2}\left(k_{x} k_{z}\right)$ satisfy the equation $\hat{H}_{0}^{2} \psi_{k_{x} k_{z} \chi}=E_{\chi}^{2}\left(k_{x} k_{z}\right) \psi_{k_{x} k_{z} \chi}$, where the operator $\hat{H}_{0}^{2}$ is given by

$$
\begin{aligned}
\hat{H}_{0}^{2}= & v_{F}^{2}\left[-\partial_{y}^{2}+\left(k_{x}-\frac{e}{c} B y \cos \eta\right)^{2}\right. \\
& \left.+\left(Q+\chi k_{z}+\frac{e}{c} \chi B y \sin \eta\right)^{2}\right] \\
& +\frac{e}{c} B v_{F}^{2}\left(\hat{\sigma}_{z} \cos \eta+\chi \hat{\sigma}_{x} \sin \eta\right) .
\end{aligned}
$$

After performing the transformation of the pseudospin basis given by Eq. (4.4) and the rotation of the coordinate frame given by Eqs. (4.2) and (4.3), the operator $\left(\hat{H}_{0}^{\prime}\right)^{2}$, given by Eq. (B2), takes the form

$$
\begin{aligned}
\left(\hat{H}_{0}^{\prime}\right)^{2}= & v_{F}^{2}\left\{-\partial_{y}^{2}+\left(k_{x^{\prime}}-\chi Q \sin \eta-\frac{e}{c} B y\right)^{2}\right. \\
& \left.+\left(k_{z^{\prime}}+\chi Q \cos \eta\right)^{2}+\frac{e}{c} B \hat{\sigma}_{z}\right\} .
\end{aligned}
$$

The operators (B2) and (B3) have the form of the Hamiltonian of a system consisting of a one-dimensional harmonic oscillator with the mass $1 / 2 v_{F}^{2}$ and the frequency $2 v_{F}^{2}|e| B / c$ and an independent spin-1/2 in a magnetic field. To obtain the eigenstates of the system, it is convenient, therefore, to introduce the ladder operators,

$$
\begin{array}{r}
\hat{a}^{\dagger}=\sqrt{\frac{c}{2|e| B}}\left(-\partial_{y}+k_{x^{\prime}}-\chi Q \sin \eta-\frac{e}{c} B y\right), \\
\hat{a}=\sqrt{\frac{c}{2|e| B}}\left(\partial_{y}+k_{x^{\prime}}-\chi Q \sin \eta-\frac{e}{c} B y\right)
\end{array}
$$

of this harmonic oscillator.

In terms of the creation and annihilation operators $\hat{a}^{\dagger}$ and $\hat{a}$, given by Eqs. (B4) and (B5), the Hamiltonian of the quasiparticles in the rotated pseudospin basis has the form

$$
\hat{H}_{0}^{\prime}=v_{F}\left(Q \cos \eta+\chi k_{z^{\prime}}\right) \hat{\sigma}_{z}+\frac{1}{\sqrt{2}} \frac{v_{F}}{l_{B}} \hat{\sigma}^{+} \hat{a}^{\dagger}+\frac{1}{\sqrt{2}} \frac{v_{F}}{l_{B}} \hat{\sigma}^{-} \hat{a} .
$$

The eigenstates and the eigenenergies of the Hamiltonian (B6), corresponding to the zeroth Landau level, are given, respectively, by Eqs. (4.8) and (4.10). 


\section{APPENDIX C: INTERVALLEY COUPLING FOR $\eta=\pi / 2$}

In this section, we present an explicit calculation of the internodal coupling $\Delta$ introduced in Sec. IV B. Because the coupling is essential at angles $\eta$ close to $\pi / 2$, we focus on $\eta=\pi / 2$, i.e., the direction of the magnetic field perpendicular to the separation between the nodes. The coupling $\Delta$ for a particular form of the function $m\left(k_{z}\right)$ has been computed microscopically in Ref. [39]. Here, we generalize this derivation to the case of a generic function $m\left(k_{z}\right)$.

Following Ref. [39], we perform a unitary rotation

$$
\psi \rightarrow \phi=e^{i \frac{\pi}{2} \hat{\sigma}_{y}} \psi
$$

in the pseudospin space and rewrite the equation $\hat{\mathcal{H}}^{2} \psi=E^{2} \psi$ for a disorder-free system in the form

$$
\begin{aligned}
& {\left[-v_{F}^{2} \partial_{y}^{2}+v_{F}^{2} k_{x}^{2}+m^{2}\left(k_{z}+\frac{e}{c} B y\right)-E^{2}\right] \phi} \\
& \quad=B m^{\prime}\left(k_{z}+\frac{e}{c} B y\right) \sigma_{z} \phi .
\end{aligned}
$$

We emphasize that near the node $\chi=-1$, the rotation $(\mathrm{C} 1)$ of the basis in the pseudospin space is distinct from that described by Eq. (4.4), which we used in Sec. IV in order to obtain the quasiparticle wave functions in the absence of the internodal coupling. Whereas the transformation (4.4) is different near different nodes, the rotation $(\mathrm{C} 1)$ is a global transformation of the basis; the two transformations match near the node with $\chi=+1$ in the case $\eta=\pi / 2$ under consideration. In this section, we also use the coordinate frame described by Eqs. (4.2) and (4.3) and shown in Fig. 3 for $\eta=\pi / 2$, i.e., with $k_{z^{\prime}}=k_{x}, k_{x^{\prime}}=-k_{z}$.

Equation (C2) is similar to the Schrödinger equation of a quadratically dispersive particle in a double-well potential
[64]. The tunnelling amplitude between the two wells may be computed in the quasiclassical approximation. To that end, we introduce the classical (complex) momentum along the $y$ axis,

$$
k_{y}(y)=\left[m^{2}\left(k_{z}+\frac{e}{c} B y\right)+v_{F}^{2} k_{x}^{2}-E^{2}\right]^{\frac{1}{2}} .
$$

The quasiclassical solutions of Eq. (C2) in the region between the minima of the wells, where the amplitude of the wave function is small, are given by

$$
\phi_{1,2}=\sum_{ \pm} C_{1,2}^{( \pm)} \frac{e^{ \pm \int_{y_{*}}^{y} k_{y} d y \pm \int_{y^{*}}^{y} \frac{d y e B m^{\prime}}{2 c k_{y}}}}{\sqrt{k_{y}}}
$$

where $C_{1}^{( \pm)}$and $C_{2}^{( \pm)}$are constants and the coordinate $y^{*}$ may be chosen arbitrarily. Between the nodes, the function $m$, which characterizes the bandwidth of the quasiparticles, is large compared to all of the other momentum scales of lowenergy quasiparticles. Therefore, in the region of applicability of Eq. (C4), it is possible to make the approximation $k_{y} \approx m$. Substituting the solutions (C4) for the wave functions between the nodes into the Schrödinger equation $\hat{H} \psi=E \psi$ with the Hamiltonian (3.1) in the absence of the impurity potential, we obtain the relations

$$
C_{2}^{( \pm)}=\left[\frac{\left(E \mp v_{F} k_{x}\right)}{2 m\left(y^{*}\right)}\right]^{ \pm 1} C_{1}^{( \pm)}
$$

between the coefficients $C_{1}^{( \pm)}$and $C_{2}^{( \pm)}$.

Equation (C4) describes the wave function between the nodes, where the exponential is small. Solving the Schrödinger equation $\hat{H} \psi=E \psi$, with the function $m$ linearized according to Eq. (4.1), gives the (non-normalized) wave function near a node of chirality $\chi$ in the form

$$
\begin{aligned}
\left(\begin{array}{l}
\phi_{1} \\
\phi_{2}
\end{array}\right)= & \cos \left[\frac{\pi c\left(E^{2}-v_{F}^{2} k_{x}^{2}\right)}{2 v_{F}^{2} e B}\right] e^{-\frac{c\left(Q+\chi k_{z}+\chi \frac{e B y}{c}\right)^{2}}{2|e| B}}\left(Q+\chi k_{z}-\chi \frac{e B y}{c}\right)^{\frac{c\left(E^{2}-v_{F}^{2} k_{x}^{2}\right)}{2 v_{F}^{2}|e| B}}\left(\begin{array}{c}
1 \\
\frac{E-\chi k_{x} c}{2 \chi\left(Q c+\chi k_{x} c-\chi B y\right)}
\end{array}\right) \\
& +\frac{\sqrt{2 \pi}}{\Gamma\left[\frac{c\left(E^{2}-v_{F}^{2} k_{x}^{2}\right)}{2 v_{F}^{2} e B}\right]}\left(\frac{|e| B}{2 c}\right)^{\frac{c\left(E^{2}-v_{F}^{2} k_{x}^{2}\right)}{2 v_{F}^{2}|e| B}+\frac{1}{2}} e^{\frac{c\left(Q+\chi k_{z}+\chi \frac{e B y}{c}\right)^{2}}{2|e| B}}\left(Q+\chi k_{z}-\chi \frac{e B y}{c}\right)^{\frac{c\left(E^{2}-v_{F}^{2} k_{x}^{2}\right)}{2 v_{F}^{2}|e| B}-1}\left(\begin{array}{c}
1 \\
\frac{2 \chi\left(Q c+\chi k_{x} c-\chi B y\right)}{E+\chi k_{x}}
\end{array}\right),
\end{aligned}
$$

where $\phi_{1}$ and $\phi_{2}$ are the components of the wave function $\phi$ with pseudospins, respectively, parallel and antiparallel to the $x$ axis. The exponent $\frac{c\left(Q+\chi k_{z}+\chi \frac{e B y}{c}\right)^{2}}{2|e| B} \equiv l_{B}^{2}\left(\chi Q+\frac{e B y}{c}-k_{x^{\prime}}\right)^{2}$ in Eq. (C6) is small near the node of chirality $\chi$, in agreement with the discussion in Sec. IV A. As Eq. (C6) applies in the vicinity of the node, where the function $m$ may be linearized, it contains both factors' contributions exponentially decaying and exponentially increasing away from the node.

Matching the asymptotics of Eq. (C6) at large values of the quantity $Q+\chi k_{z}+\chi \frac{e B y}{c}$ with the solution $(\mathrm{C} 4)$ between the nodes and obtaining the values of the coefficients $C_{1}$ and $C_{2}$, we arrive at the values of the quasiparticle energies in the form

$$
\frac{\left(E^{2}-v_{F}^{2} k_{x}^{2}\right) c}{2|e| B v_{F}^{2}}=\frac{1}{2 \pi} e^{-\frac{2 c}{|e| B v_{F}} \int_{-Q}^{Q} m\left(p_{z}\right) d p_{z}},
$$

which leads to the value of the internodal coupling $\Delta$ given by Eq. (4.15) in the main text.

\section{Weakly hybridized states}

At $\eta=\pi / 2$, the dispersion of the quasiparticles at decoupled nodes is given by $E=\chi v_{F} k_{x}$, as follows from Eq. (4.10). The internodal coupling leads to the hybridization of quasiparticle states at different nodes and the modification of the dispersion. As discussed in Sec. IV B, the states at $\eta=\pi / 2$ get hybridized most strongly for small momenta $k_{x}$.

As we also discuss in Sec. VI, the chemical potential $\mu$ in realistic WSMs corresponds to large momenta, $\left|k_{x}\right| \gg \Delta / v_{F}$, at which the states are weakly hybridized by the internodal tunnelling. Despite being weak, taking into account this hybridization is essential for obtaining a finite conductivity in the case of the angle $\eta$ being close to the $\pi / 2$ that we consider. 
In the regime under consideration, a state with a positive momentum $k_{x}$ and energy $E \approx v_{F} k_{x}+\frac{\Delta^{2}}{2 v_{F} k_{x}}$ [cf. Eq. (4.14)] at the node $\chi=+1$ acquires, due to hybridization, a small correction from the other node, $\chi=-1$. The wave function of such a state is given by

$$
\begin{aligned}
\left|\phi_{+1 \mathbf{k}}\right\rangle= & \left(\frac{|e| B}{\pi c}\right)^{1 / 4} e^{\left.-\frac{c\left(Q+k_{z}+\frac{e B y}{c}\right.}{2|e| B}\right)^{2}}\left(\begin{array}{l}
1 \\
0
\end{array}\right) \\
& -\sqrt{\frac{E-v_{F} k_{x}}{E+v_{F} k_{x}}}\left\{\left(\frac{|e| B}{\pi c}\right)^{1 / 4} e^{\left.-\frac{c\left(Q-k_{z}-\frac{e B y}{c}\right.}{2|e| B}\right)^{2}}\left(\begin{array}{c}
\frac{E+v_{F} k_{x}}{2 m} \\
1
\end{array}\right)\right. \\
& \left.\left.-\left(\frac{\pi c}{|e| B}\right)^{1 / 4}\left(\frac{E}{v_{F}}+k_{x}\right) e^{\frac{c\left(Q-k_{2}-\frac{e B y}{C}\right.}{2|e| B}}\left(\begin{array}{l}
1 \\
0
\end{array}\right)\right\} . \text { (C } 8\right)
\end{aligned}
$$

The first term in the right-hand side of Eq. (C8) describes the wave function of the quasiparticle at node $\chi=1$. This term is peaked at $k_{z}=-Q-\frac{e B y}{c}$, i.e., at the location of the first node in the $x z$ plane in momentum space, as discussed in Sec. IV A. The last two terms in Eq. (C8) describe the small correction due to the presence of the other node, with the chirality $\chi=-1$. Those terms are peaked at $k_{z}=Q-$ $\frac{e B y}{c}$ and are suppressed by the small prefactor $\sqrt{\frac{E-v_{F} k_{x}}{E+v_{F} k_{x}}} \approx$ $\frac{\Delta}{v k_{x} \sqrt{2}}$ in the limit of large momenta $k_{x} \gg \Delta / v_{F}$ under consideration.

Similarly, the state at the node with chirality $\chi=-1$ is weakly hybridized due to the internodal tunnelling, with the other node whose chirality is $\chi=-1$. The wave function of this state is given by

$$
\begin{aligned}
& \left|\phi_{-1 \mathbf{k}}\right\rangle=\left(\frac{|e| B}{\pi c}\right)^{1 / 4} e^{-\frac{c\left(Q-k_{z}-\frac{e B y}{c}\right)^{2}}{2|e| B}}\left(\begin{array}{l}
0 \\
1
\end{array}\right) \\
& -\sqrt{\frac{E+v_{F} k_{x}}{E-v_{F} k_{x}}}\left\{\left(\frac{|e| B}{\pi c}\right)^{1 / 4} e^{-\frac{c\left(Q+k_{z}+\frac{e B y}{C}\right)^{2}}{|e| B}}\left(\begin{array}{c}
1 \\
\frac{E-v_{F} k_{x}}{2 m}
\end{array}\right)\right. \\
& \left.-\left(\frac{\pi c}{|e| B}\right)^{1 / 4}\left(\frac{E}{v_{F}}-k_{x}\right) e^{\frac{c\left(Q+k_{z}+\frac{e B y}{C}\right)^{2}}{2 e|e|}}\left(\begin{array}{l}
0 \\
1
\end{array}\right)\right\} .
\end{aligned}
$$

The energy of this state is given by $E \approx-v_{F} k_{x}-\frac{\Delta^{2}}{2 v_{F} k_{x}}$ for the large momenta $k_{x}$ under consideration. The first and the last two terms in Eq. (C9) describe, respectively, the state of a particle at the node with chirality $\chi=-1$ and a correction due to the presence of the other node.

\section{APPENDIX D: INTERNODAL SCATTERING RATE AWAY $\operatorname{FROM} \eta=\pi / 2$}

In this section, we present details of the calculation of the internodal scattering rate (5.3) for angles $\eta$ away from $\pi / 2$, where the effect of coherent internodal tunnelling on the quasiparticle dispersion and scattering may be neglected. For simplicity, we assume that the system is rectangular, with the edges parallel to the $x^{\prime}, y$, and $z^{\prime}$ axes.

As discussed in Sec. V, the scattering rate is determined by the matrix element of scattering of a quasiparticle near node $\chi$ with momentum $\mathbf{p}=\left(\mathbf{p}_{x^{\prime}}, \mathbf{p}_{z^{\prime}}\right)$ in the $x^{\prime} z$ plane into the state with momentum $\mathbf{k}=\left(\mathbf{k}_{x^{\prime}}, \mathbf{k}_{z^{\prime}}\right)$ near node $\chi^{\prime}$ on the potential $u\left(\mathbf{r}-\mathbf{R}_{i}\right)$ of the $i$ th impurity, with the location $\mathbf{R}_{i}=$ $\left(R_{x}^{(i)}, R_{y}^{(i)}, R_{z}^{(i)}\right)$, given by

$$
\left\langle\psi_{\chi \mathbf{p}}\left|u\left(\mathbf{r}-\mathbf{R}_{i}\right)\right| \psi_{\chi^{\prime} \mathbf{k}}\right\rangle \equiv \int d \mathbf{r} \psi_{\chi \mathbf{p}}^{\dagger}(\mathbf{r}) u\left(\mathbf{r}-\mathbf{R}_{i}\right) \psi_{\chi^{\prime} \mathbf{k}}(\mathbf{r})=\frac{4 \pi Z_{i} e^{2}}{V \varepsilon} \int d \mathbf{r} \psi_{\chi}^{\dagger}(\mathbf{r}) \psi_{\chi^{\prime}}(\mathbf{r}) \sum_{\mathbf{q}} \frac{e^{i \mathbf{q}\left(\mathbf{r}-\mathbf{R}_{i}\right)}}{q^{2}+\varkappa^{2}},
$$

where $\psi_{\chi \mathbf{p}}(\mathbf{r})$ and $\psi_{\chi^{\prime} \mathbf{k}}(\mathbf{r})$ are the wave functions of the respective states, given by Eq. (4.9). In Eq. (D1), we used the Fourier transform $u(\mathbf{q})=\frac{4 \pi Z_{i} e^{2}}{q^{2}+\varkappa^{2}}$ of the potential [55] of an impurity.

The conductivity is determined by internodal scattering, with $\chi=-\chi^{\prime}$, while intranodal scattering, corresponding to $\chi=\chi^{\prime}$, has no effect on transport since it does not change the quasiparticle velocity. Using Eq. (4.9) for the states with $\chi=1$ and $\chi^{\prime}=-1$ gives the matrix element for internodal scattering in the form

$$
\begin{aligned}
\left\langle\psi_{+1 \mathbf{p}}\left|u\left(\mathbf{r}-\mathbf{R}_{i}\right)\right| \psi_{-1 \mathbf{k}}\right\rangle= & \frac{4 \pi Z_{i} e^{2} \cos \eta}{V \varepsilon} \sum_{q_{y}} \exp \left[-\frac{c\left(p_{x^{\prime}}-k_{x^{\prime}}-2 Q \sin \eta\right)^{2}}{4|e| B}-\frac{c q_{y}^{2}}{4|e| B}+\frac{i c q_{y}}{e B}\left(\frac{p_{x^{\prime}}+k_{x^{\prime}}}{2}-\frac{e B R_{y}^{(i)}}{c}\right)\right] \\
& \times \frac{e^{-i\left(p_{x^{\prime}}-k_{x^{\prime}}\right) R_{x^{\prime}}^{(i)}-i\left(p_{z^{\prime}}-k_{z^{\prime}}\right) R_{z^{\prime}}^{(i)}}}{q_{y}^{2}+\left(p_{x^{\prime}}-k_{x^{\prime}}\right)^{2}+\left(p_{z^{\prime}}-k_{z^{\prime}}\right)^{2}+\varkappa^{2}} .
\end{aligned}
$$

Performing disorder averaging, $\langle\ldots\rangle_{\text {dis }}=\frac{1}{V} \int \ldots d \mathbf{R}_{i}$, of the square of the matrix element over the location of the impurity gives

$$
\left\langle\left|\left\langle\psi_{+1 \mathbf{p}}|U| \psi_{-1 \mathbf{k}}\right\rangle\right|^{2}\right\rangle_{\text {dis }}=N\left(\frac{4 \pi e^{2} \cos \eta}{V \varepsilon}\right)^{2} e^{-\frac{\left(\frac{c\left(p_{x^{\prime}}-k_{x^{\prime}}-2 Q \sin \eta\right)^{2}}{2|e| B}\right.}{2}} \sum_{q_{y}} \frac{e^{-\frac{c q_{y}^{2}}{2|e| B}}}{\left[q_{y}^{2}+\left(p_{x^{\prime}}-k_{x^{\prime}}\right)^{2}+\left(p_{z^{\prime}}-k_{z^{\prime}}\right)^{2}+\varkappa^{2}\right]^{2}},
$$

where $N$ is the number of impurities in the system.

The expression in the denominator in Eq. (D3) may be approximated as

$$
q_{y}^{2}+\left(p_{x^{\prime}}-k_{x^{\prime}}\right)^{2}+\left(p_{z^{\prime}}-k_{z^{\prime}}\right)^{2}+\varkappa^{2} \approx(2 Q)^{2} .
$$

Indeed, the sum with respect to $q_{y}$ in Eq. (D3) is dominated by momenta $q_{y}$ of the order of the inverse magnetic length $l_{B}^{-1}$, which is given by Eq. (3.4) and is significantly smaller than the separation $2 Q$ between the nodes. The inverse screening length $\varkappa$ is of the order of $\alpha^{\frac{1}{2}} / l_{B}$ and is also significantly 
exceeded by the momentum $Q$. Because the energies of the quasiparticles are small compared to the bandwidth and according to Eq. (4.10), $p_{z^{\prime}} \approx-Q \cos \eta$ and $k_{z^{\prime}} \approx Q \cos \eta$. The dynamics of the quasiparticles correspond to the range of momenta where the function $m$ in the Hamiltonian (3.1) may be linearized, as given by Eq. (4.1). Together with Eqs. (4.2) and (4.3), this gives $k_{x^{\prime}} \approx Q \sin \eta$ and $p_{x^{\prime}} \approx-Q \sin \eta$. Taking into account the values of all momenta and neglecting all momentum scales smaller than $Q$, we arrive at the approximation (D4).

Performing the integration with respect to momentum $q_{y}$ (with the replacement $\sum_{q_{y}} \rightarrow L_{y} \int \frac{d q_{y}}{2 \pi}$ ) and utilizing Eq. (D3) gives

$$
\begin{aligned}
& \left\langle\left|\left\langle\psi_{+1 \mathbf{p}}|U| \psi_{-1 \mathbf{k}}\right\rangle\right|^{2}\right\rangle_{\mathrm{dis}} \\
& \approx N\left(\frac{4 \pi e^{2} \cos \eta}{V \varepsilon}\right)^{2} e^{-\frac{c\left(p_{x^{\prime}}-k_{x^{\prime}}-2 Q \sin \eta\right)^{2}}{2|e| B}} \frac{L_{y}}{2 \pi} \sqrt{\frac{2|e| B \pi}{c}} \frac{1}{\left[4 Q^{2}\right]^{2}} .
\end{aligned}
$$

Substituting the disorder-averaged scattering element (D5) into the expression (5.1) and performing integration over the momentum components $k_{x^{\prime}}$ and $k_{z^{\prime}}$, we arrive at the expression (5.3) for the internodal scattering rate.

\section{APPENDIX E: INTERNODAL SCATTERING RATE AT $\eta=\pi / 2$}

In this section, we provide the details of the calculation of the quasiparticle scattering rate $1 / \tau$ at $\eta=\pi / 2$, i.e., for the magnetic field perpendicular to the separation between the nodes. The resistivity at this angle is determined by the processes of scattering between states $\left|\phi_{+1}\right\rangle$ and $\left|\phi_{-1 \mathbf{k}}\right\rangle$ given, respectively, by Eqs. (C8) and (C9). The scattering rate between such states is given by

$$
\frac{1}{\tau}=2 \pi \int\left\langle\left|\left\langle\phi_{+1 \mathbf{p}}|U| \phi_{-1 \mathbf{k}}\right\rangle\right|^{2}\right\rangle_{\mathrm{dis}} \delta\left(E_{+1 \mathbf{p}}-E_{-1 \mathbf{k}}\right) \frac{S_{x z} d \mathbf{p}}{(2 \pi)^{2}},
$$

where $E_{+1 \mathbf{p}}$ and $E_{-1 \mathbf{k}}$ are, respectively, the energies of the states $\left|\phi_{+1 \mathbf{p}}\right\rangle$ and $\left|\phi_{-1 \mathbf{k}}\right\rangle$ and $S_{x z}$ is the cross-section area of the system in the $x z$ plane.

Similarly to the case of internodal scattering at angles $\eta$ away from $\pi / 2$, the scattering rate $1 / \tau$ is determined by the matrix element $\left\langle\phi_{+1 \mathbf{p}}|| u\left(\mathbf{r}-\mathbf{R}_{i}\right)|| \phi_{-1 \mathbf{k}}\right\rangle$ of scattering off an impurity at location $\mathbf{R}_{i}$. Noticing that the exponentials in Eqs. (C8) and (C9) which grow away from the nodes do not contribute to the matrix elements, we obtain

$$
\begin{aligned}
\left\langle\phi_{+1}|\mathbf{p}| u\left(\mathbf{r}-\mathbf{R}_{i}\right) \mid \phi_{-1 \mathbf{k}}\right\rangle= & -e^{-\frac{c\left(p_{z}-k_{z}\right)^{2}}{4|e| B}} \sqrt{\frac{E_{-1 \mathbf{k}}+v_{F} k_{x}}{E_{-1 \mathbf{k}}-v_{F} k_{x}}} \frac{4 \pi Z_{i} e^{2}}{V \varepsilon} \sum_{q_{y}} \frac{e^{-\frac{c q_{y}^{2}}{4|e| B}-\frac{i c q_{y}}{2 e B}\left(2 Q+p_{z}+k_{z}\right)-i q_{y} R_{y}^{(i)}-i\left(p_{x}-k_{x}\right) R_{x}^{(i)}-i\left(p_{z}-k_{z}\right) R_{z}^{(i)}}}{q_{y}^{2}+\left(p_{x}-k_{x}\right)^{2}+\left(p_{z}-k_{z}\right)^{2}+\varkappa^{2}} \\
& -e^{-\frac{c\left(p_{z}-k_{z}\right)^{2}}{4|e| B}} \sqrt{\frac{E_{+1 \mathbf{p}}-v_{F} p_{x}}{E_{+1 \mathbf{p}}+v_{F} p_{x}}} \frac{4 \pi Z_{i} e^{2}}{V \varepsilon} \sum_{q_{y}} \frac{e^{-\frac{c q_{y}^{2}}{4|e| B}-\frac{i c q_{y}}{2 e B}\left(-2 Q+p_{z}+k_{z}\right)-i q_{y} R_{y}^{(i)}-i\left(p_{x}-k_{x}\right) R_{x}^{(i)}-i\left(p_{z}-k_{z}\right) R_{z}^{(i)}}}{q_{y}^{2}+\left(p_{x}-k_{x}\right)^{2}+\left(p_{z}-k_{z}\right)^{2}+\varkappa^{2}} .
\end{aligned}
$$

The first and the second lines in Eq. (E2) come from the wave functions near nodes $\chi=-1$ and $\chi=+1$.

The matrix element of scattering off the total potential $U(\mathbf{r})=\sum_{i} u\left(\mathbf{r}-\mathbf{R}_{i}\right)$, averaged over the realizations of the potential, is given by

$$
\begin{aligned}
\left\langle\left|\left\langle\phi_{+1 \mathbf{p}}|U| \phi_{-1 \mathbf{k}}\right\rangle\right|^{2}\right\rangle_{\mathrm{dis}}= & \frac{N}{V}\left\langle\left|\left\langle\phi_{+1 \mathbf{p}}\left|u\left(\mathbf{r}-\mathbf{R}_{i}\right)\right| \phi_{-1 \mathbf{k}}\right\rangle\right|^{2}\right\rangle_{\mathrm{dis}} \\
= & N\left(\frac{4 \pi e^{2}}{V \varepsilon}\right)^{2} \frac{L_{y}}{2 \pi}\left\{\left(\frac{E_{-1 \mathbf{k}}+v_{F} k_{x}}{E_{-1 \mathbf{k}}-v_{F} k_{x}}+\frac{E_{+1 \mathbf{p}}-v_{F} p_{x}}{E_{+1 \mathbf{p}}+v_{F} p_{x}}\right) \int \frac{d q_{y} e^{-\frac{c\left(p_{z}-k_{z}\right)^{2}}{2|e| B}-\frac{c q_{y}^{2}}{2|e| B}}}{\left[q_{y}^{2}+\left(p_{x}-k_{x}\right)^{2}+\left(p_{z}-k_{z}\right)^{2}+\varkappa^{2}\right]^{2}}\right. \\
& \left.+\sqrt{\frac{\left(E_{-1 \mathbf{k}}+v_{F} k_{x}\right)\left(E_{+1 \mathbf{p}}-v_{F} p_{x}\right)}{\left(E_{-1 \mathbf{k}}-v_{F} k_{x}\right)\left(E_{+1 \mathbf{p}}+v_{F} p_{x}\right)}} \int \frac{d q_{y} e^{-\frac{c\left(p_{z}-k_{z}\right)^{2}}{2|e| B}-\frac{c q_{y}^{2}}{2|e| B}}\left(e^{\frac{2 i Q c q_{y}}{e B}}+e^{-\frac{2 i Q c q_{y}}{e B}}\right)}{\left[q_{y}^{2}+\left(p_{x}-k_{x}\right)^{2}+\left(p_{z}-k_{z}\right)^{2}+\varkappa^{2}\right]^{2}}\right\} .
\end{aligned}
$$

The quantities $\left[q_{y}^{2}+\left(p_{x}-k_{x}\right)^{2}+\left(p_{z}-k_{z}\right)^{2}+\varkappa^{2}\right]^{\frac{1}{2}}$ in Eq. (E3) have the meaning of the effective momentum change of a plane-wave state scattered off the potential. We emphasize, however, that unlike the case of internodal scattering considered in Appendix E, this difference cannot be approximated by the separation $2 Q$ between the Weyl nodes.

To compute the scattering time (E1), we first perform the integration of the expression (E3) with respect to the momentum $p_{z}$. The first line gives a contribution proportional to

$$
\int \frac{d p_{z} d q_{y} e^{-\frac{c\left(p_{z}-k_{z}\right)^{2}}{2|e| B}-\frac{c q_{y}^{2}}{2|e| B}}}{\left[q_{y}^{2}+\left(p_{x}-k_{x}\right)^{2}+\left(p_{z}-k_{z}\right)^{2}+\varkappa^{2}\right]^{2}}=\frac{\pi}{\left(p_{x}-k_{x}\right)^{2}+\varkappa^{2}}-\frac{\pi c}{2|e| B} e^{\frac{c\left(p_{x}-k x\right)^{2}}{2|e| B}+\frac{c \varkappa^{2}}{2|e| B}} E_{1}\left[\frac{c\left(p_{x}-k_{x}\right)^{2}}{2|e| B}+\frac{c \varkappa^{2}}{2|e| B}\right],
$$

where $E_{1}(x)=\int_{1}^{\infty} d t e^{-t x} / t$ is the exponential integral. The characteristic momentum difference $p_{x}-k_{x}$ is of the order of $|\mu| / v_{F}$, with $\mu$ being the chemical potential, and in the limit of strong magnetic fields under consideration (the "ultraquantum" limit) is exceeded by the inverse magnetic length $l_{B}^{-1}=\sqrt{|e| B / c}$. As a result and also considering the smallness of the magnetic length $l_{B}$ in comparison with the screening length $\varkappa$, the second term in the right-hand side of Eq. (E4) may be neglected and we 
may approximate

$$
\int \frac{d p_{z} d q_{y} e^{-\frac{c\left(p_{z}-k_{z}\right)^{2}}{2|e| B}-\frac{c q_{y}^{2}}{2|e| B}}}{\left[q_{y}^{2}+\left(p_{x}-k_{x}\right)^{2}+\left(p_{z}-k_{z}\right)^{2}+\varkappa^{2}\right]^{2}} \approx \frac{\pi}{\left(p_{x}-k_{x}\right)^{2}+\varkappa^{2}} .
$$

The integral of the second line of Eq. (E3) with respect to the momentum $p_{z}$ may be represented in the form

$$
\int \frac{d p_{z} d q_{y} e^{-\frac{c\left(p_{z}-k_{z}\right)^{2}}{2|e| B}-\frac{c q_{y}^{2}}{2|e| B}}\left(e^{\frac{2 i Q c q_{y}}{e B}}+e^{-\frac{2 i Q c q_{y}}{e B}}\right)}{\left[q_{y}^{2}+\left(p_{x}-k_{x}\right)^{2}+\left(p_{z}-k_{z}\right)^{2}+\varkappa^{2}\right]^{2}}=\frac{2 \pi I\left[l_{B} Q, l_{B} \sqrt{\left(p_{x}-k_{x}\right)^{2}+\varkappa^{2}}\right]}{\left(p_{x}-k_{x}\right)^{2}+\varkappa^{2}},
$$

where the magnetic length $l_{B}$ is given by Eq. (3.4) and the integral $I(s, t)$ is given by Eq. (6.3). Performing integration with respect to $p_{z}$ in the expression (E1) for the scattering rate and utilizing Eqs. (E5)-(E6), we arrive at the scattering rate given by Eqs. (6.2) and (6.3).

In the main text, we consider the ranges of parameters that correspond to two limiting cases in the integral $I(s, t)$,

$$
I(s, t)=\frac{t^{2}}{\pi} \int \frac{\cos (2 s y) e^{-\frac{z^{2}+y^{2}}{2}}}{\left(y^{2}+z^{2}+t^{2}\right)^{2}} d z d y \rightarrow \begin{cases}1, & s t \ll 1 \\ 0, & s t \gg 1 .\end{cases}
$$

[1] X. Wan, A. M. Turner, A. Vishwanath, and S. Y. Savrasov, Topological semimetal and Fermi-arc surface states in the electronic structure of pyrochlore iridates, Phys. Rev. B 83, 205101 (2011).

[2] S.-Y. Xu, I. Belopolski, N. Alidoust, M. Neupane, G. Bian, C. Zhang, R. Sankar, G. Chang, Z. Yuan, C.-C. Lee, S.-M. Huang, H. Zheng, J. Ma, D. S. Sanchez, B. Wang, A. Bansil, F. Chou, P. P. Shibayev, H. Lin, S. Jia, and M. Z. Hasan, Discovery of a Weyl fermion semimetal and topological Fermi arcs, Science 349, 613 (2015).

[3] B. Q. Lv, H. M. Weng, B. B. Fu, X. P. Wang, H. Miao, J. Ma, P. Richard, X. C. Huang, L. X. Zhao, G. F. Chen, Z. Fang, X. Dai, T. Qian, and H. Ding, Experimental Discovery of Weyl Semimetal TaAs, Phys. Rev. X 5, 031013 (2015).

[4] B. Q. Lv, N. Xu, H. M. Weng, J. Z. Ma, P. Richard, X. C. Huang, L. X. Zhao, G. F. Chen, C. E. Matt, F. Bisti, V. N. Strocov, J. Mesot, Z. Fang, X. Dai, T. Qian, M. Shi, and H. Ding, Observation of Weyl nodes in TaAs, Nat. Phys. 11, 724 (2015).

[5] S.-Y. Xu, I. Belopolski, D. S. Sanchez, C. Zhang, G. Chang, C. Guo, G. Bian, Z. Yuan, H. Lu, T.-R. Chang, P. P. Shibayev, M. L. Prokopovych, N. Alidoust, H. Zheng, C.-C. Lee, S.-M. Huang, R. Sankar, F. Chou, C.-H. Hsu, H.-T. Jeng, A. Bansil, T. Neupert, V. N. Strocov, H. Lin, S. Jia, and M. Z. Hasan, Experimental discovery of a topological Weyl semimetal state in TaP, Sci. Adv. 1, e1501092 (2015).

[6] S.-Y. Xu, N. Alidoust, I. Belopolski, Z. Yuan, G. Bian, T.-R. Chang, H. Zheng, V. N. Strocov, D. S. Sanchez, G. Chang, C. Zhang, D. Mou, Y. Wu, L. Huang, C.-C. Lee, S.-M. Huang, B. Wang, A. Bansil, H.-T. Jeng, T. Neupert, A. Kaminski, H. Lin, S. Jia, and M. Zahid Hasan, Discovery of a Weyl fermion state with Fermi arcs in niobium arsenide, Nat. Phys. 11, 748 (2015).

[7] L. X. Yang, Z. K. Liu, Y. Sun, H. Peng, H. F. Yang, T. Zhang, B. Zhou, Y. Zhang, Y. F. Guo, M. Rahn, D. Prabhakaran, Z. Hussain, S.-K. Mo, C. Felser, B. Yan, and Y. L. Chen, Weyl semimetal phase in the non-centrosymmetric compound TaAs, Nat. Phys. 11, 728 (2015).
[8] N. Xu, H. M. Weng, B. Q. Lv, C. E. Matt, J. Park, F. Bisti, V. N. Strocov, D. Gawryluk, E. Pomjakushina, K. Conder, N. C. Plumb, M. Radovic, G. AutGIos, O. V. Yazyev, Z. Fang, X. Dai, T. Qian, J. Mesot, H. Ding, and M. Shi, Observation of Weyl nodes and Fermi arcs in tantalum phosphide, Nat. Commun. 7, 11006 (2016).

[9] M. Z. Hasan, S.-Y. Xu, I. Belopolski, and S.-M. Huang, Discovery of Weyl fermion semimetals and topological Fermi arc states, Annu. Rev. Condens. Matter Phys. 8, 289 (2017).

[10] N. P. Armitage, E. J. Mele, and A. Vishwanath, Weyl and Dirac semimetals in three-dimensional solids, Rev. Mod. Phys. 90, 015001 (2018).

[11] O. Vafek and A. Vishwanath, Dirac fermions in solids: From high- $T_{c}$ cuprates and graphene to topological insulators and Weyl semimetals, Annu. Rev. Condens. Matter Phys. 5, 83 (2014).

[12] A. A. Burkov, Weyl metals, Annu. Rev. Condens. Matter Phys. 9, 359 (2018).

[13] A. A. Burkov, Chiral anomaly and transport in Weyl metals, J. Phys.: Condens. Matter 27, 113201 (2015).

[14] D. T. Son and B. Z. Spivak, Chiral anomaly and classical negative magnetoresistance of Weyl metals, Phys. Rev. B 88, 104412 (2013).

[15] H. B. Nielsen and M. Ninomiya, Absence of neutrinos on a lattice: (i). Proof by homotopy theory, Nucl. Phys. B 185, 20 (1981).

[16] D. E. Kharzeev and H.-U. Yee, Anomaly induced chiral magnetic current in a Weyl semimetal: Chiral electronics, Phys. Rev. B 88, 115119 (2013).

[17] X. Huang, L. Zhao, Y. Long, P. Wang, D. Chen, Z. Yang, H. Liang, M. Xue, H. Weng, Z. Fang, X. Dai, and G. Chen, Observation of the Chiral-Anomaly-Induced Negative Magnetoresistance in 3D Weyl Semimetal TaAs, Phys. Rev. X 5, 031023 (2015).

[18] C.-L. Zhang, S.-Y. Xu, I. Belopolski, Z. Yuan, Z. Lin, B. Tong, G. Bian, N. Alidoust, C.-C. Lee, S.-M. Huang, T.-R. Chang, 
G. Chang, C.-H. Hsu, H.-T. Jeng, M. Neupane, D. S. Sanchez, H. Zheng, J. Wang, H. Lin, C. Zhang, H.-Z. Lu, S.-Q. Shen, T. Neupert, M. Zahid Hasan, and S. Jia, Signatures of the Adler-Bell-Jackiw anomaly in a Weyl fermion semimetal, Nat. Commun. 7, 10735 (2016).

[19] X. Yang, Y. Liu, Z. Wang, Y. Zheng, and Z.-a. Xu, Chiral anomaly induced negative magnetoresistance in topological Weyl semimetal NbAs, arXiv:1506.03190.

[20] J. Du, H. Wang, Q. Chen, Q. Mao, R. Khan, B. Xu, Y. Zhou, Y. Zhang, J. Yang, B. Chen, C. Feng, and M. Fang, Large unsaturated positive and negative magnetoresistance in Weyl semimetal TaP, Sci. China Phys. Mech. Astron. 59, 657406 (2016).

[21] Z. Wang, Y. Zheng, Z. Shen, Y. Lu, H. Fang, F. Sheng, Y. Zhou, X. Yang, Y. Li, C. Feng, and Z.-A. Xu, Helicity-protected ultrahigh mobility Weyl fermions in NbP, Phys. Rev. B 93, 121112(R) (2016).

[22] S. Jia, S.-Y. Xu, and M. Z. Hasan, Weyl semimetals, Fermi arcs and chiral anomalies, Nat. Mater. 15, 1140 (2016).

[23] J. Xiong, S. K. Kushwaha, T. Liang, J. W. Krizan, M. Hirschberger, W. Wang, R. J. Cava, and N. P. Ong, Evidence for the chiral anomaly in the Dirac semimetal $\mathrm{Na}_{3} \mathrm{Bi}$, Science 350, 413 (2015).

[24] M. Hirschberger, S. Kushwaha, Z. Wang, Q. Gibson, S. Liang, C. A. Belvin, B. A. Bernevig, R. J. Cava, and N. P. Ong, The chiral anomaly and thermopower of Weyl fermions in the halfheusler GdPtBi, Nat. Mater. 15, 1161 (2016).

[25] S. Liang, J. Lin, S. Kushwaha, J. Xing, N. Ni, R. J. Cava, and N. P. Ong, Experimental Tests of the Chiral Anomaly Magnetoresistance in the Dirac-Weyl Semimetals $\mathrm{Na}_{3} \mathrm{Bi}$ and GdPtBi, Phys. Rev. X 8, 031002 (2018).

[26] P. J. W. Moll, N. L. Nair, T. Helm, A. C. Potter, I. Kimchi, A. Vishwanath, and J. G. Analytis, Transport evidence for Fermiarc-mediated chirality transfer in the Dirac semimetal $\mathrm{Cd}_{3} \mathrm{As}_{2}$, Nature (London) 535, 266 (2016).

[27] A. C. Potter, I. Kimchi, and A. Vishwanath, Quantum oscillations from surface Fermi arcs in Weyl and Dirac semimetals, Nat. Commun. 5, 5161 (2014).

[28] S. V. Syzranov, Y. I. Rodionov, and B. Skinner, Adiabatic dechiralization and thermodynamics of Weyl semimetals, Phys. Rev. B 98, 081114(R) (2018).

[29] E. V. Gorbar, V. A. Miransky, I. A. Shovkovy, and P. O. Sukhachov, Consistent hydrodynamic theory of chiral electrons in Weyl semimetals, Phys. Rev. B 97, 121105(R) (2018).

[30] E. V. Gorbar, V. A. Miransky, I. A. Shovkovy, and P. O. Sukhachov, Hydrodynamic electron flow in a Weyl semimetal slab: Role of Chern-Simons terms, Phys. Rev. B 97, 205119 (2018).

[31] A. Lucas, R. A. Davison, and S. Sachdev, Hydrodynamic theory of thermoelectric transport and negative magnetoresistance in Weyl semimetals, Proc. Natl. Acad. Sci. 113, 9463 (2016).

[32] N. Yamamoto, Chiral transport of neutrinos in supernovae: Neutrino-induced fluid helicity and helical plasma instability, Phys. Rev. D 93, 065017 (2016).

[33] V. Galitski, M. Kargarian, and S. Syzranov, Dynamo Effect and Turbulence in Hydrodynamic Weyl Metals, Phys. Rev. Lett. 121, 176603 (2018).

[34] E. V. Gorbar, V. A. Miransky, I. A. Shovkovy, and P. O. Sukhachov, Nonlocal transport in Weyl semimetals in the hydrodynamic regime, Phys. Rev. B 98, 035121 (2018).
[35] P. O. Sukhachov, E. V. Gorbar, I. A. Shovkovy, and V. A. Miransky, Collective excitations in Weyl semimetals in the hydrodynamic regime, J. Phys.: Condens. Matter 30, 275601 (2018).

[36] J. Gooth, F. Menges, N. Kumar, V. Suß, C. Shekhar, Y. Sun, U. Drechsler, R. Zierold, C. Felser, and B. Gotsmann, Thermal and electrical signatures of a hydrodynamic electron fluid in tungsten diphosphide, Nat. Commun. 9, 4093 (2018).

[37] P. Kim, J. H. Ryoo, and C.-H. Park, Breakdown of the Chiral Anomaly in Weyl Semimetals in a Strong Magnetic Field, Phys. Rev. Lett. 119, 266401 (2017).

[38] C.-K. Chan and P. A. Lee, Emergence of gapped bulk and metallic side walls in the zeroth Landau level in Dirac and Weyl semimetals, Phys. Rev. B 96, 195143 (2017).

[39] D. R. Saykin, K. S. Tikhonov, and Y. I. Rodionov, Landau levels with magnetic tunneling in a Weyl semimetal and magnetoconductance of a ballistic $p-n$ junction, Phys. Rev. B 97, 041202(R) (2018).

[40] M.-C. Hsu, H. Lin, M. Zahid Hasan, and S.-M. Huang, Topologically distinct Weyl-fermion pairs: On the effect of magnetic tunnelling, arXiv:1903.07484.

[41] Y. Jiang, Z. Dun, S. Moon, H. Zhou, M. Koshino, D. Smirnov, and Z. Jiang, Landau quantization in coupled Weyl points: A case study of semimetal NbP, Nano Lett. 18, 7726 (2018).

[42] C.-L. Zhang, S.-Y. Xu, C. M. Wang, Z. Lin, Z. Z. Du, C. Guo, C.-C. Lee, H. Lu, Y. Feng, S.-M. Huang, G. Chang, C.-H. Hsu, H. Liu, H. Lin, L. Li, C. Zhang, J. Zhang, X.-C. Xie, T. Neupert, M. Z. Hasan, H.-Z. Lu, J. Wang, and S. Jia, Magnetictunnelling-induced Weyl node annihilation in TaP, Nat. Phys. 13, 979 (2017).

[43] H.-Z. Lu and S.-Q. Shen, Quantum transport in topological semimetals under magnetic fields, Frontiers Phys. 12, 127201 (2017).

[44] P. Goswami, J. H. Pixley, and S. Das Sarma, Axial anomaly and longitudinal magnetoresistance of a generic three-dimensional metal, Phys. Rev. B 92, 075205 (2015).

[45] J. Behrends, R. Ilan, and J. H. Bardarson, Anomalous conductance scaling in strained Weyl semimetals, Phys. Rev. Res. 1, 032028(R) (2019).

[46] X. Xiao, K. T. Law, and P. A. Lee, Magnetoconductivity in Weyl semimetals: Effect of chemical potential and temperature, Phys. Rev. B 96, 165101 (2017).

[47] R. D. dos Reis, M. O. Ajeesh, N. Kumar, F. Arnold, C. Shekhar, M. Naumann, M. Schmidt, M. Nicklas, and E. Hassinger, On the search for the chiral anomaly in Weyl semimetals: The negative longitudinal magnetoresistance, New J. Phys. 18, 085006 (2016).

[48] J.-P. Jay-Gerin, M. J. Aubin, and L. G. Caron, The electron mobility and the static dielectric constant of $\mathrm{Cd}_{3} \mathrm{As}_{2}$ at $4.2 \mathrm{~K}$, Solid State Commun. 21, 771 (1977).

[49] Cadmium arsenide $\left(\mathrm{Cd}_{3} \mathrm{As}_{2}\right)$ optical properties, dielectric constants, in Non-Tetrahedrally Bonded Elements and Binary Compounds I, edited by O. Madelung, U. Rössler, and M. Schulz (Springer, Berlin, 1998), p. 1.

[50] G. S. Jenkins, C. Lane, B. Barbiellini, A. B. Sushkov, R. L. Carey, F. Liu, J. W. Krizan, S. K. Kushwaha, Q. Gibson, T.-R. Chang, H.-T. Jeng, H. Lin, R. J. Cava, A. Bansil, and H. D. Drew, Three-dimensional Dirac cone carrier dynamics in $\mathrm{Na}_{3} \mathrm{Bi}$ and $\mathrm{Cd}_{3} \mathrm{As}_{2}$, Phys. Rev. B 94, 085121 (2016). 
[51] J. Buckeridge, D. Jevdokimovs, C. R. A. Catlow, and A. A. Sokol, Bulk electronic, elastic, structural, and dielectric properties of the Weyl semimetal TaAs, Phys. Rev. B 93, 125205 (2016).

[52] The value of the chirality assigned to each node here matches the flux $\chi=\frac{1}{2 \pi} \int \mathcal{B}(\mathbf{k}) d S_{\mathbf{k}}$ of the field $\mathcal{B}=-i \nabla_{\mathbf{k}} \times$ $\left\langle\psi_{+}(\mathbf{k})\left|\nabla_{\mathbf{k}}\right| \psi_{+}(\mathbf{k})\right\rangle$ (measured in units of $2 \pi$ ) through a surface in momentum space surrounding the node, where $\left|\psi_{+}(\mathbf{k})\right\rangle$ is the state of a quasiparticle with momentum $\mathbf{k}$ in the conduction band of a disorder-free semimetal in zero magnetic field.

[53] Y. I. Rodionov and S. V. Syzranov, Conductivity of a Weyl semimetal with donor and acceptor impurities, Phys. Rev. B 91, 195107 (2015).

[54] B. Skinner, Coulomb disorder in three-dimensional Dirac systems, Phys. Rev. B 90, 060202(R) (2014).

[55] Hereafter, the potentials $U(\mathbf{r})$ and $u(\mathbf{r})$ are the potential energies of the quasiparticles and not electrostatic potentials.

[56] A. A. Abrikosov, Fundamentals of the Theory of Metals (Elsevier, Oxford, 1988).

[57] V. F. Gantmakher, Electrons and Disorder in Solids (Oxford University Press, Oxford, 2005).

[58] J. Behrends and J. H. Bardarson, Strongly angledependent magnetoresistance in Weyl semimetals with long-range disorder, Phys. Rev. B 96, 060201(R) (2017).

[59] S. Jeon, B. B. Zhou, A. Gyenis, B. E. Feldman, I. Kimchi, A. C. Potter, Q. D. Gibson, R. J. Cava, A. Vishwanath, and A. Yazdani, Landau quantization and quasiparticle interference in the three-dimensional Dirac semimetal $\mathrm{Cd}_{3} \mathrm{As}_{2}$, Nat. Mater. 13, 851 (2014).

[60] W. Shi, B. J. Wieder, H. L. Meyerheim, Y. Sun, Y. Zhang, Y. Li, L. Shen, Y. Qi, L. Yang, J. Jena, P. Werner, K. Koepernik, S. Parkin, Y. Chen, C. Felser, B. A. Bernevig, and Z. Wang, A charge-density-wave Weyl semimetal, arXiv:1909.04037.

[61] A. Cortijo, Y. Ferreirós, K. Landsteiner, and M. A. H. Vozmediano, Elastic Gauge Fields in Weyl Semimetals, Phys. Rev. Lett. 115, 177202 (2015).

[62] D. I. Pikulin, A. Chen, and M. Franz, Chiral Anomaly from Strain-Induced Gauge Fields in Dirac and Weyl Semimetals, Phys. Rev. X 6, 041021 (2016).

[63] A. Cortijo, D. Kharzeev, K. Landsteiner, and M. A. H. Vozmediano, Strain-induced chiral magnetic effect in Weyl semimetals, Phys. Rev. B 94, 241405(R) (2016).

[64] L. D. Landau and E. M. Lifshitz, Quantum Mechanics: NonRelativistic Theory, Course of Theoretical Physics (Elsevier Science, New York, 1981). 